223

Munir H. Nayfeh

10.2.1 Plasma Model - 224

10.2.2 Miniaturized Metal: Subwavelength Concentration of Light - 226

10.2.3 Miniaturization-Induced Coloration of Metals - 229

10.2.4 Plasmonic Lenses - 230

10.2.5 Metamaterials: Negative Refractive Index - 232

10.2.6 Heat Loss: Are Plasmonic-Based Devices Practical? - 233

10.3.1 Bandgap and Exciton - 233

10.3.2 Direct and Indirect Bandgap Materials - 234

10.3.3 Enhancing and Blue Shifting of Luminescence by Quantum Confinement - 235

10.3.4 Making Silicon Glow: Quantum Confinement - 236

10.3.5 Optical Nonlinearity in Nanosilicon - 239

10.3.6 Optical Gain in Nanosilicon-Based Material - 241

10.4.1 Integration of Optics and Electronics - 241

10.4.2 Confined Light in Service of Substance Detection - 242

10.4.3 Nanofabrication and Nanolithography - 244

10.4.4 Photovoltaics and Photocurrent -246

10.4.5 Solid State LED White Lighting - 248

10.4.6 Plasmonic Hyperthermic-Based Treatment and Monitoring of Acute Disease - 250

M.H. Nayfeh ( $\bowtie)$

Department of Physics, University of Illinois at Urbana-Champaign, 1110 W. Green Street, Urbana, IL 61801, USA

e-mail: m-nayfeh@illinois.edu

○ The Authors) 2016

M.D. Al-Amri et al. (eds.), Optics in Our Time, DOI 10.1007/978-3-319-31903-2_10 


\subsection{Introduction}

Optics is one of the most important branches of physics. It involves the study of the behavior and properties of light in vacuum as well as the study of its interactions with matter in the gas, liquid, and solid states [1]. Moreover, the field encompasses the construction of instruments that use or detect light that may serve many other fields, over a wide range of the electromagnetic waves from UV to infrared light.

Nanotechnology or nanoscience and technology, on the other hand, aims at construction, understanding, and putting to use ultrasmall particles [2-4]. Miniaturization of all types of matter including dielectrics, metals, semiconductors, polymers, etc., affords interesting novel properties, especially optical properties. The novel size regime is intermediate between the largest molecules and $100 \mathrm{~nm}$. In this regime, phenomenon may not be as predictable as those observed at larger scales. Using nanoparticles as building blocks to construct advanced devices that exploit their novel properties is at the heart of nanotechnology.

It is to be noted that naturally existing colloids, micelles, polymer molecules, and phase-separated regions in block copolymers, for example, fall in this size regime. More recently, naturally unknown but interesting classes of nanostructures such as carbon nanotubes [5], silicon nanoparticles [6, 7], metal nanoparticles and nanorods $[8,9]$, and compound semiconductor quantum dots [10] have been designed and fabricated. The application of such extremely small particles can find applications across all fields $[4,11]$. In this article we will focus on how miniaturization down to the nanoscale regime impacts the behavior, properties, and interactions of light with matter, especially metals and semiconductors, and how it enables novel advanced devices with application in electronics, photonics, energy and lighting, and biomedicine.

\subsection{Optics in Nanometals: Nature of Interaction of Light with Metal}

In a dielectric, all electrons are bound in atoms; and each atom interacts with light individually through the interaction of a single bound electron. The total effect in a sample is simply the sum of the individual atomic responses. In a metal solid, some electrons are bound to atoms and others are not bound in specific atoms or ions. Upon interaction with light, two processes can take place in metal. In interband transition, bound electrons, i.e., electrons in the valence band can be promoted due to light absorption to an empty level in the conduction band where they become not bound to a specific ion. Interband absorption forms a significant loss mechanism in metal at optical frequencies. On the other hand, in a metal solid, electrons that are already in the conduction band form a see or cloud of electrons not bound to specific atoms or ions. The cloud can interact with and move under an external electric force collectively at the same time.

\subsubsection{Plasma Model}

- Figure 10.1 (left) shows a schematic of the lattice of bulk metal where electrons are subjected to a force created by the electric field of light. Free electrons move opposite to the direction of the electric field while positive ions are stationary, thus causing a shift between the center of distribution of the negative charge and the positive charge. In the absence of the force the centers of the two distributions coincide. - Figure 10.1 (right) shows the same situation for a very small sphere of metal, namely a nanoparticle. 


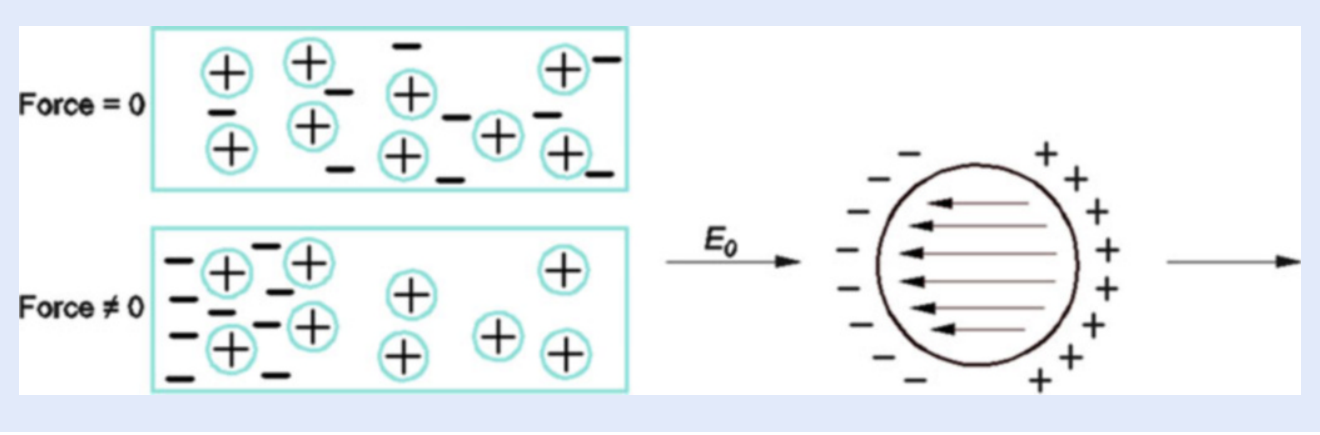

- Fig. 10.1 Sketch of metal lattice subjected to a force created by an electric field. (Left) bulk and (Right) nanoparticle

Electrons in a small sphere are described by a simplified model of a gas of free electrons that moves against a fixed background of positive ion cores [12-14]. This has been called a plasma model. In the model, details of the lattice potential and electron-electron interactions are not included explicitly; rather they are simply incorporated into the effective optical mass $m$ of each electron. Under irradiation, the negative electron's see or cloud gets pushed back and forth relative to the fixed background of positive ion at the frequency of oscillation in the electric field of the electromagnetic light wave. In addition, the cloud experiences an effective drag force due to collisions with the positive ion core which tends to slow it down. According to the plasma model, electrons oscillate and their motion is damped with a characteristic collision frequency $\gamma$ as high as $10^{14}$ per second $(100 \mathrm{THz})$ (corresponding to the frequency of infrared light). The time elapsed between two consecutive collisions, $\tau=1 / \gamma=10^{-14} \mathrm{~s}$, is known as the relaxation time of the free electron gas. The balance of these two forces gives the response or what is called the real part of the dielectric function of the cloud, $\epsilon(\omega)$, to the light wave:

$$
\varepsilon(\omega)=1-\frac{n e^{2}}{\varepsilon_{0} m \omega^{2}}
$$

Where $n$ is the density of electrons in the cloud, $e$ and $m$ are the charge and the effective mass of a single of electron, $\epsilon_{0}$ is the dielectric function of vacuum, and $\omega$ is the frequency of the incoming light wave. It is customary to define a useful quantity called plasma frequency or plasmon frequency $\omega_{\mathrm{p}}$ in terms of the following group of constants:

$$
\omega_{p}=\sqrt{\frac{n e^{2}}{\varepsilon_{0} m}}
$$

In terms of the plasma frequency, the dielectric function takes the simple form $1-\omega^{2} / \omega_{\mathrm{p}}^{2}$. For a metal with a free electron density of $10^{23} / \mathrm{cm}^{3}, \omega_{\mathrm{p}}$ corresponds to the frequency of ultraviolet light (plasmon energy $\hbar \omega_{\mathrm{p}} \sim 10 \mathrm{eV}$ ) which is 100 or even 1000 times larger than the relaxation frequency $\gamma$.

- Figure 10.2 plots $\epsilon(\omega)$ as a function of frequency. At resonance with the plasma frequency $\left(\omega=\omega_{\mathrm{p}}\right), \epsilon(\omega)$ drops to zero. This is a plasma oscillation resonance. For light with a frequency below the plasma frequency, the dielectric function is negative and the light cannot penetrate the sample, rather it is totally reflected. Above the plasma frequency the light waves penetrate the sample as sketched in the figure. 

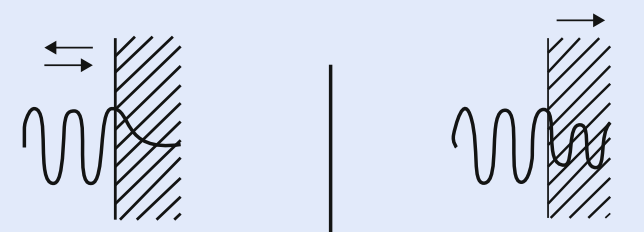

Total reflection

$\varepsilon(\omega)$

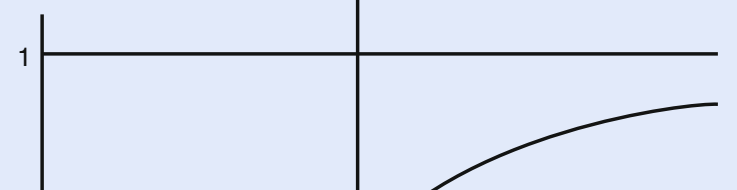

Fig. 10.2 Plot of the dielectric function $\epsilon(\omega)$ as a function of frequency for a metal. $\epsilon(\omega)$ drops to zero at resonance with the plasma frequency $\left(\omega=\omega_{\mathrm{p}}\right.$ ) (Image from $>$ https://www. coursehero.com/file/10546609/Plasmonics/)

\subsubsection{Miniaturized Metal: Subwavelength Concentration of Light}

We now discuss how the interaction of metal with light manifest itself as we reduce the size of the metal sample from large bulk samples to small nanoparticles. We will compare three kinds of structures sketched in - Fig. 10.3: bulk, which is labeled as three-dimensional (3D); a sheet or quantum well, which is labeled as two-dimensional (2D); quantum wire, which is labeled as one-dimensional (1D); and nanoparticle or quantum dot, which is labeled as zero-dimensional (0D).

\section{Bulk Material (3D)}

Because free conduction electrons can move over very large distances in bulk metal objects, electrons on average do not oscillate against a certain localized ions with a restoring force. Instead the motion is actually similar to a mass being dragged in a viscous fluid. If the light has a frequency above the plasma frequency (in the ultraviolet (UV) range for metals), electrons will not even oscillate and the light will simply be transmitted or absorbed in interband transitions of the metal. If the light has a frequency below UV, electrons will oscillate out of phase with the incident light, causing a strong reflection. At the plasma frequency, the dielectric function is 0 .

\section{Thin Film or Sheet (2D)}

When bulk metal is shrunk to a thin film of a few nm thicknesses, electrons cannot move very far in the direction normal to the film. In this case there will be electron oscillations upon light exposure; but they will only exist at the surface of the film. 


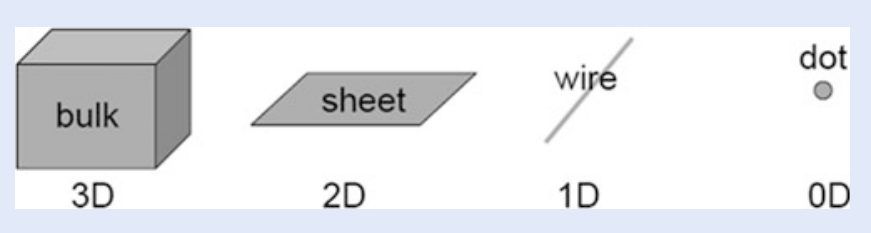

- Fig. 10.3 Schematic of four kinds of structures: bulk, a sheet or quantum well, quantum wire, and nanoparticle or quantum dot

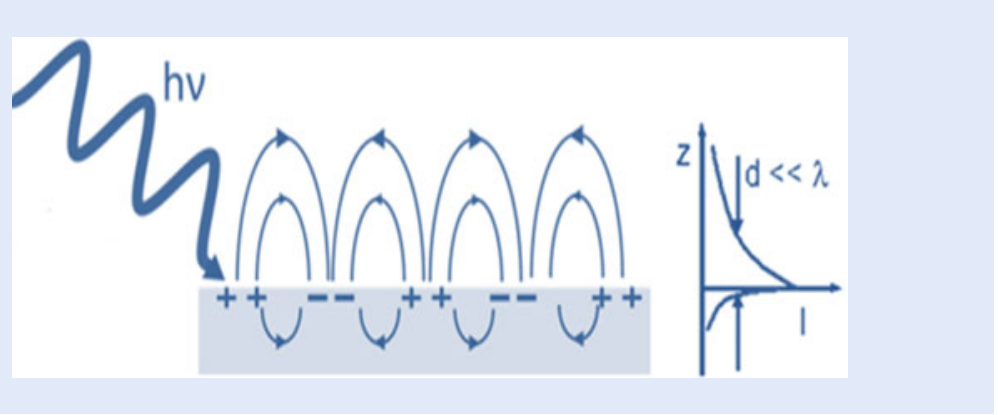

- Fig. 10.4 Schematic of light interaction with a few $\mathrm{nm}$ thin metal films (left) shows electron oscillations at the surface of the film, accompanied with propagation of charge waves along the film. (Adapted from $>$ https://en.wikipedia.org/wiki/Surface_plasmon). (Right) Sketch of the field intensity with distance from the surface

This is accompanied with propagation of charge waves along the film. This is known as surface plasmon polaritons (SPPs) as sketched in - Fig. 10.4 (left). In fact, detailed Maxwell's theory shows that the surface waves can propagate along the surface with a broad spectrum of frequencies from 0 up to $\omega_{\mathrm{p}} / \sqrt{2} 2$, where $\omega_{\mathrm{p}}$ is the plasma frequency defined above. At $\omega_{\mathrm{p}} / \sqrt{ } 2$ the dielectric function is -1 with a charge-wave wavelength shorter than the wavelength of the incident light. The combined effect is a mixed or hybrid light-electron-wave-state. This results in intense light-matter interactions with unprecedented optical response. As shown in - Fig. 10.4 (right), the intense optical field resulting from the hybrid field is a local one, extending outside the thin film into the dielectric only few nanometers, a distance much less than the wavelength of the incident light $(d$ « $\lambda)$. Thus visible light, which has a wavelength of approximately half a micrometer, can be concentrated by a factor of nearly 100 to travel through metal films just a few nanometers (nm) thick.

\section{Nanowire (1D)}

A variety of metal nanowires have been fabricated and studied. They may come as solid nanowire of metal with a surrounding dielectric jacket, i.e., glass or air. For example, chemically prepared silver nanowires $\sim 100 \mathrm{~nm}$ diameters were fabricated [15]. The wires were found to support surface plasmon modes propagating along the wires. The wavelength of the propagating charge wave is shortened to about half the wavelength of exciting light. The propagation length of SPP is about $10 \mu \mathrm{m}$. The reflectivity at both ends of the wire is about $25 \%$. Those characteristics are sufficient for the wires to be used as an optical instrument, namely surface plasmon Fabry-Perot resonators. Wires can also be in the form of a cylindrical shell of metal. The shell may have an inner dielectric jacket, such as glass (silicon oxide). The bore of the system may be filled with a semiconductor, such as $\mathrm{Si}$ or CdSe. More complicated variations of these architectures have been fabricated to accommodate ports for light entry or extraction. Semiconductor wires with 
hemispheres of metal on both ends have also been fabricated. The schemes involving hybrid semiconductor-plasmonic (metal) architectures will be discussed in - Sect. 10.4.1 on integrating optics with electronics.

\section{Nanoparticles/Dot (OD)}

Bulk is now shrunk in three dimensions to form particles, such that the dimension of the particle is less than the wavelength of incident light. Because the size of the particle is small compared to the wavelength the incident electric field will be constant across the nanoparticle, inducing a uniform displacement of the electron density, making the electron motion in phase (collective cloud motion). Because electrons are confined within the small particle, they will oscillate while being accompanied by a strong restoring force from specific positive ionic core background (• Fig. 10.5a). The restoring force leads to non-propagating (also called evanescent) collective oscillation of the surface cloud with a characteristic oscillation frequency similar to a simple harmonic oscillator. This is unlike bulk which does not have a specific oscillation frequency. - Figure 10.5b shows representative field lines, effectively resembling those of an oscillating electric dipole. Another

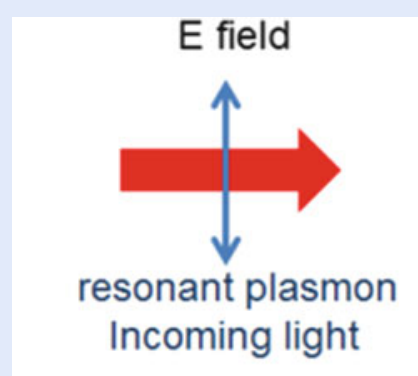

(a)
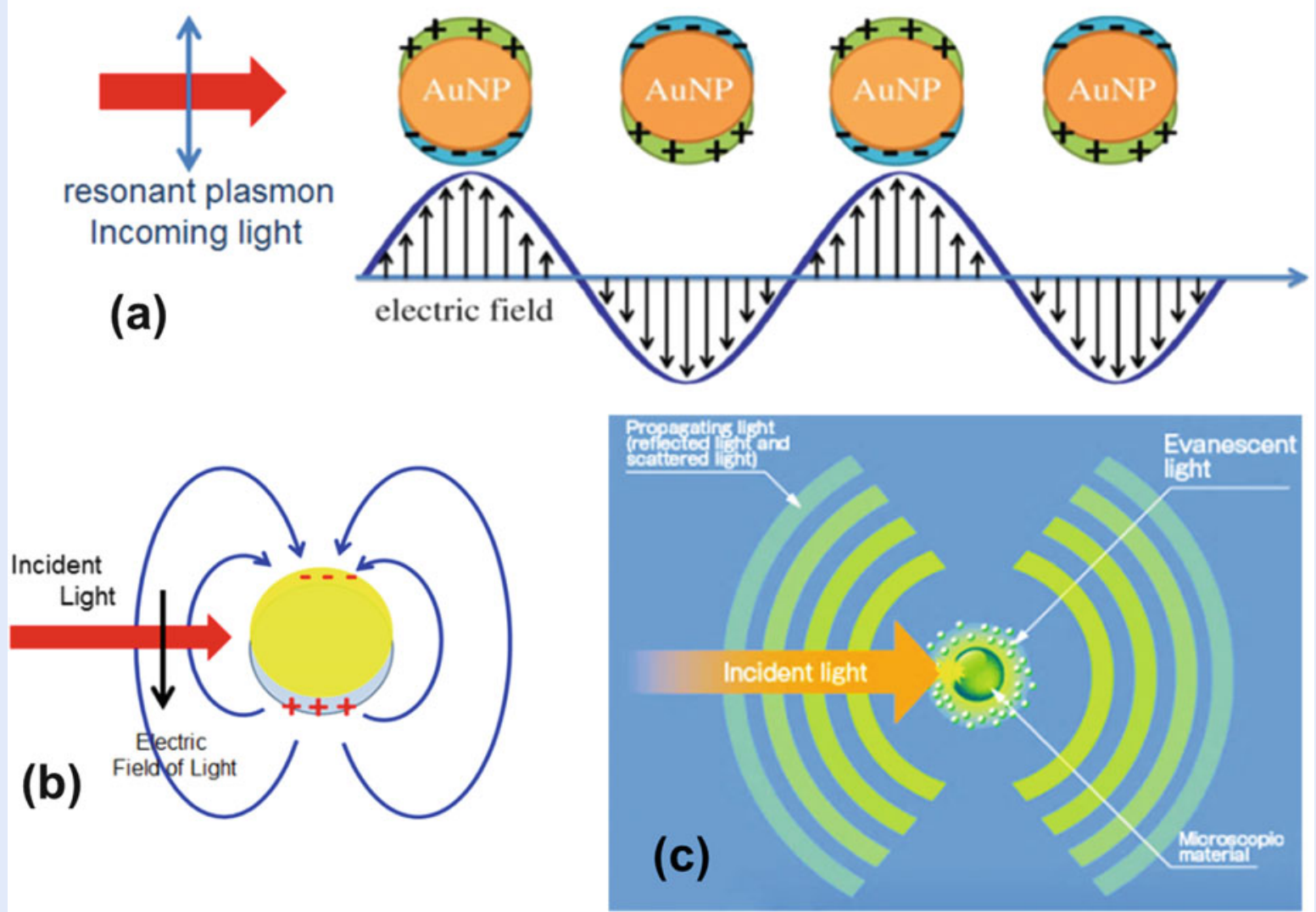

- Fig. 10.5 Schematic of the interaction of a gold nanoparticle with linearly polarized light beam. (a) Electrons oscillate while being accompanied by a strong restoring force from specific positive ionic core background (Adapted from E. Yasun, H. Kang, H. Erdal, S. Cansiz, I. Ocsoy, Y-F. Huang, W. Tan, Cancer cell sensing and therapy using affinity tag-conjugated gold nanorods, Interface Focus 3 (2013) 0006: > http://dx.doi.org/ 10.1098/rsfs.2013.0006). (b) Representative field lines, effectively resembling those of an oscillating electric dipole. (c) Region where the non-propagating (evanescent) light is localized, related only to the size of the nanoparticle, not to the wavelength (Image adapted from [16]) 
striking effect is that the collective oscillations lead to a large absorption and scattering cross section, as well as an amplified local optical electromagnetic field. The electric field has its maximum strength just outside the nanoparticle and drops rapidly with distance extending only to $\sim 30 \mathrm{~nm}$. For small particles less than $\sim 15 \mathrm{~nm}$, light absorption dominates; whereas for nanoparticles greater than $\sim 15 \mathrm{~nm}$ scattering dominates. In fact, detailed Maxwell's theory shows that surface waves can be resonantly sustained with frequency at $\omega_{\mathrm{p}} / \sqrt{3}$, which corresponds to dielectric function of -2 . We should stress because the evanescent light is non-propagating it is localized within the approximate radius of the particle, even if the size of that particle is much smaller than the incident light wavelength (- Fig. 10.5c). The region where the non-propagating evanescent light is localized is related only to the object's size and not to the wavelength, which can be considered as light with no diffraction limit. The nanoparticle practically confines light into super intense "hot-spot."

\subsubsection{Miniaturization-Induced Coloration of Metals}

Figure 10.6 (top left) shows a large block of gold under illumination by white light. When light strikes the large block, the red and green components are reflected; while the blue component is absorbed and scattered. Interband transitions of bulk gold give it a yellow color. But a metallic luster is also added

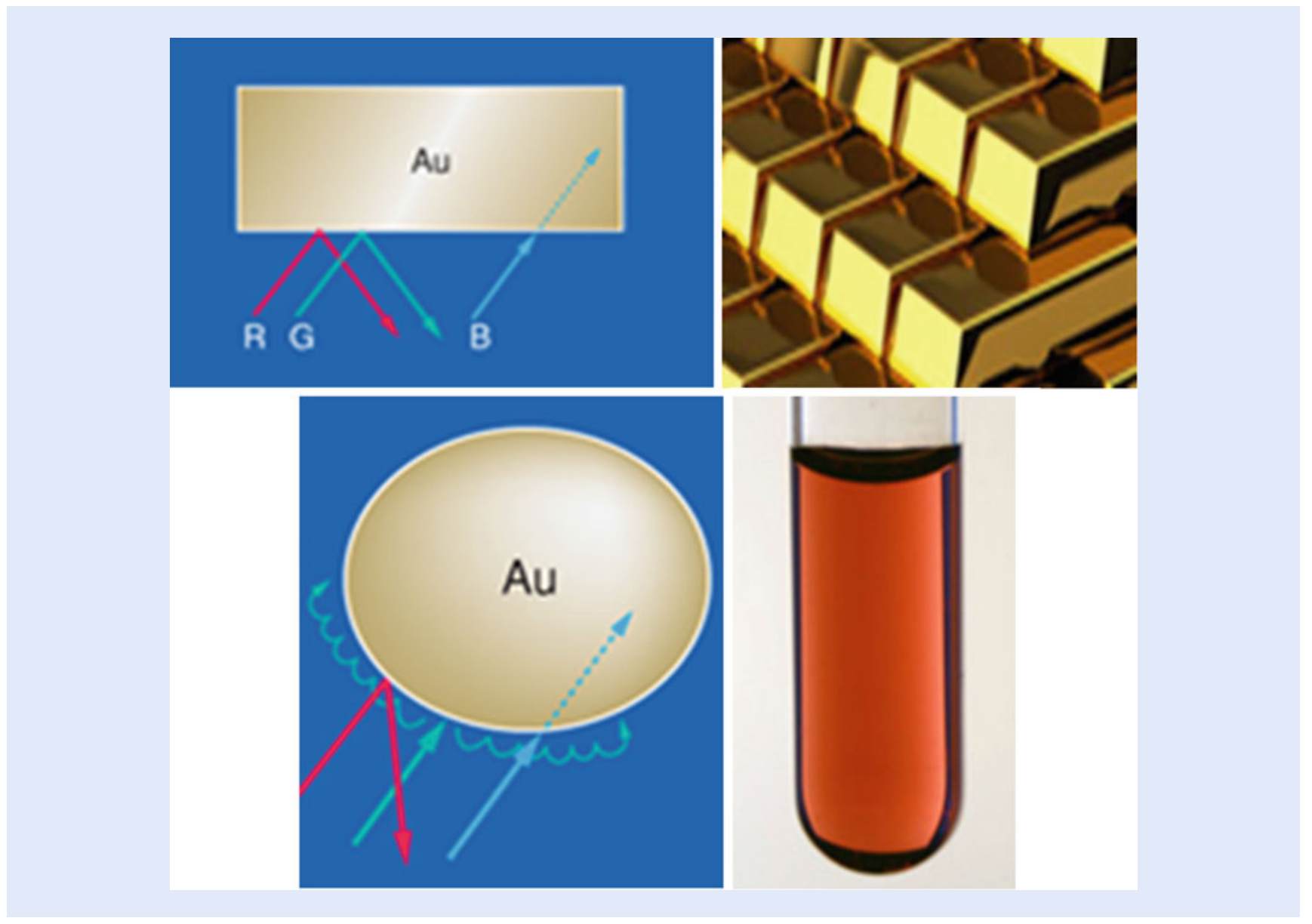

Fig. 10.6 Schematic of the interaction of gold with white light, showing the individual responses to red, green, and blue (RGB) components, as well as the resulting color as seen by the naked eye (top row) large block of gold and (bottom row) colloid of gold nanoparticles. (Adapted from [16]) 
to the yellow color, which appears as a golden color (• Fig. 10.6 top right). Charge oscillations in bulk do not have a specific frequency to cause specific coloration.

When white light strikes, on the other hand, the nanoparticle shown in - Fig. 10.6 (bottom left), the color will be different. This is due to a specific plasmon resonance due to a confined collective oscillation of electrons. A plasmon resonance in gold nanoparticles occurs over a thin slice of light at a frequency in the green. Thus not all of the components of incoming white light resonate and are absorbed. Among the RGB components, only the G (green) component resonates with the electrons and is absorbed in the gold nanoparticle. The B (blue) light is absorbed and scattered, and only the remaining $\mathrm{R}$ (red) component gets reflected or passes through. This is the reason that stained glass mixed with gold nanoparticles appears red to the naked eye. - Figure 10.6 (bottom right) demonstrates that, when light strikes a gold nanoparticle colloid, only the red color is reflected or passes through [16].

\subsubsection{Plasmonic Lenses}

Having a negative refractive index is the basic principle behind plasmonic lenses. This is a very common property for noble metal at specific frequency. There are two types of such lenses. One type is based on confinement enhancement. Another is based on transmission enhancement of evanescent waves. In this section we present examples of metal-based lenses.

\section{Confinement-Based Lensing}

We discuss two configurations for confinement-based lensing: a continuous conic waveguide concentrator and discontinuous chain of nanoindentation (particles). (1) Consider the hollow cone of metal shown in $\bullet$ Fig. 10.7 (left). The radius of the cone gradually decreases from $50 \mathrm{~nm}$ to $2 \mathrm{~nm}$, for example. When light strikes the cone and a plasmonic resonance is excited at the opening of the cone, a surface plasmonic polariton (SPP) propagates towards the tip. This causes accumulation of energy resulting in a giant local field at the tip, as shown in - Fig. 10.7 (left). - Figure 10.7 (right) displays the amplitudes of the local optical field in the cross section of the cone for the normal and longitudinal (with respect to the axis) components of the optical electric field. The magnitude of the field grows significantly as the oscillations approach the tip. The transverse $\mathrm{x}$ component grows by an order of magnitude, while the longitudinal $z$ component, which is very small far from the tip, grows relatively much stronger at the tip. This causes the local field to increase by nearly 3 orders of magnitude in intensity and four orders in energy

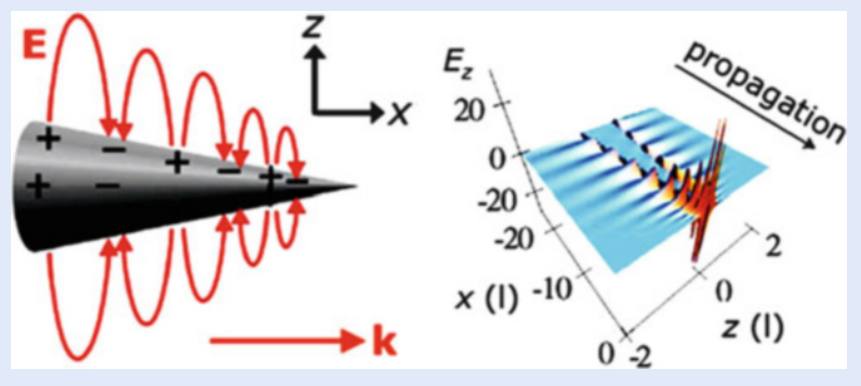

Fig. 10.7 (Left) Geometry of a conic nanoplasmonic waveguide showing propagation of a charge oscillation wave. (Right) Snapshot of instantaneous $E_{z}$ fields in the longitudinal cross section ( $x z$ ) plane, normalized to far-zone (excitation) field. (Adapted from "Stockman MI (2004) Phy Rev Lett 93: 137404”) 

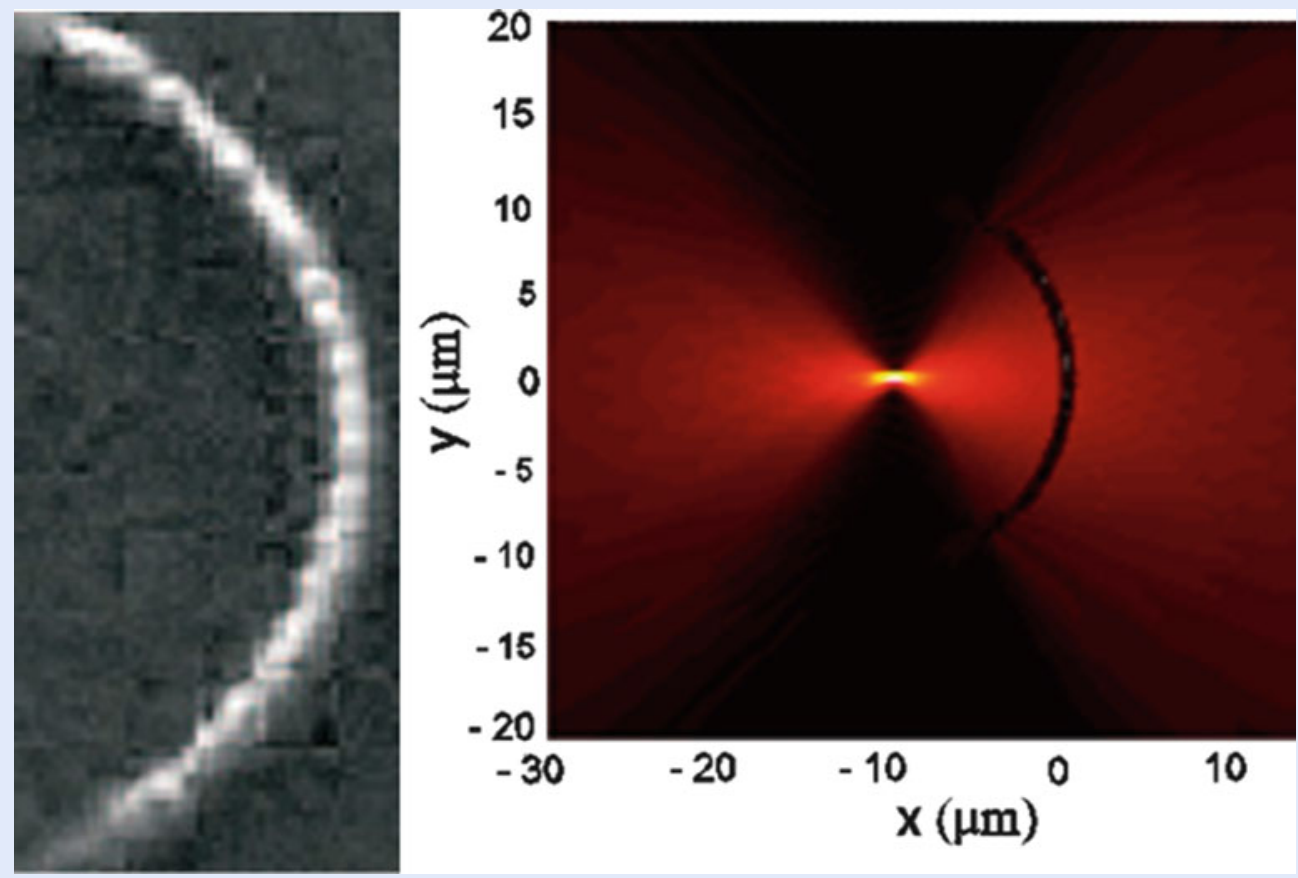

- Fig. 10.8 Focusing of light by a curved chain of spheroid gold nanoparticles indented in a thin gold film (left) top view image of the chain using a scanning electron microscope (SEM), (right) Magnitude of scattered electric field calculated above the gold surface. The illuminating beam has a wavelength of $800 \mathrm{~nm}$ and is incident perpendicular to the gold surface and polarized along $x$-direction (Adapted from "Evlyukhin AB, et al. (2007) Opt Exp 15: 16667-16680")

density. It should be noted that the propagation is slowed down and asymptotically stopped when it approaches the tip. It never actually reaches the tip (the travel time to the tip is logarithmically divergent) [17]. The cone as such may represent a tapered plasmonic waveguide. (2) Consider a chain of nanoparticles shown in - Fig. 10.8 (left). Fabrication starts with a thin gold film. Nanoindentations (nanoparticles) are made on the film in a parabolic chain configuration. The radius of curvature of the chain is $10 \mu \mathrm{m}$. The particle diameter and inter-particle distance are $\sim 350$ and $850 \mathrm{~nm}$, respectively, while the particle height is $300 \mathrm{~nm}$. When light, in the wavelength range of 700-860 nm, for example, is normally incident on the thin film on the right of the chain, charge oscillations are induced on the surface of the film which travel or propagate to the left towards the chain (surface plasmon polariton or SPP). When the waves hit the gold nanoparticles they get focused to a submicron spot, as shown in • Fig. 10.8 (right) [18].

\section{Transmission-Based Lensing}

Another concept of lensing involves transmission through holes or slits. An array of holes is drilled in an opaque metallic film in an arc formation, as shown in - Fig. 10.9a. The diameter of the holes is of nanoscale. For a plane wave incident upon such a structure, the phase shift experienced by light as it passes through each individual hole is sensitive to either the length, width, or even the materials inside the hole. With the adjustment of the properties of individual holes, it becomes possible to achieve a focusing action [19].

A slit-based lens is built by first depositing a $400 \mathrm{~nm}$ thick flat gold film on silicon oxide substrate. Then an ion beam is used to mill 13 rectangular slits in the thin film, as shown in the left panel of $\bullet$ Fig. 10.9b. The slits increase in widths from $80 \mathrm{~nm}$ at the center slit to $150 \mathrm{~nm}$ on the left or right end. - Figure 10.9c 


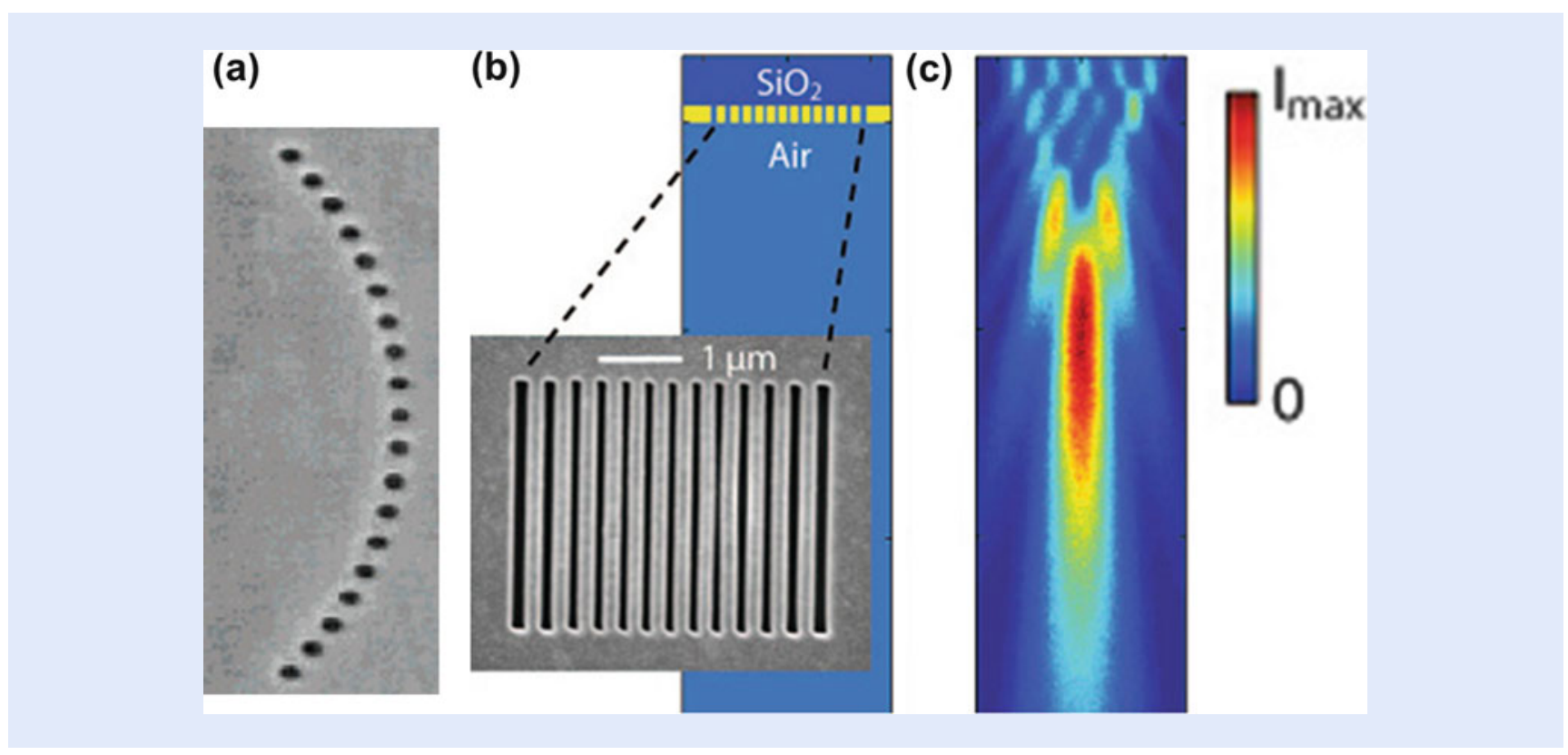

- Fig. 10.9 Focusing of light by transmission through holes or slits. (a) Chain of holes in a metal plate. (b) Nanoscale slit array drilled in gold film on a fused silica substrate (dark blue). The film is $400 \mathrm{~nm}$ thick (yellow). The air slits are different in widths (80-150 nm) (light blue). The inset shows a scanning electron micrograph of the structure as viewed from the air-side. (C) Focusing pattern measured by confocal scanning optical microscopy (CSOM) (Adapted from “Lieven Verslegers et al., Nano Lett. 9, 235-238 (2009)")

gives the measured field intensity in a cross section through the center of the slits (along the $x$-direction). The measurement demonstrates focusing of the wave. Thus this configuration acts as a far-field cylindrical lens for light at optical frequencies [20].

\subsubsection{Metamaterials: Negative Refractive Index}

The above discussion shows that metal dielectric interface, with features smaller than the wavelength of light separated by distances smaller than the wavelength of light, is very special. At plasmon resonances they have a negative index. In fact those may be considered as a class of more general material called metamaterials that exhibit properties beyond those found in nature. In 1967 [21] Victor Veselago theorized that material with negative refractive index would exhibit optical properties opposite to those of dielectrics, such as glass or air. Contrary to dielectrics, when light propagates in metamaterial (1) light refracts on the other side of the normal to the interface, that is, energy is transported in a direction opposite to the dielectric case, as shown in - Fig. 10.10; (2) light produces negative pressure, which pulls metamaterial towards it instead of positive pressure which pushes away as in conventional material. The basic principle behind all of the opposite effects is closely related to the above plasmonic effect. They are due to the collective interaction of the light with the electron clouds at the surface of the conductor. This photon-plasmon interaction generates intense, localized optical fields. The waves are confined to the interface between metal and insulator. This narrow channel serves as a transformative guide that, in effect, traps and compresses the wavelength of incoming light to a fraction of its original value. 


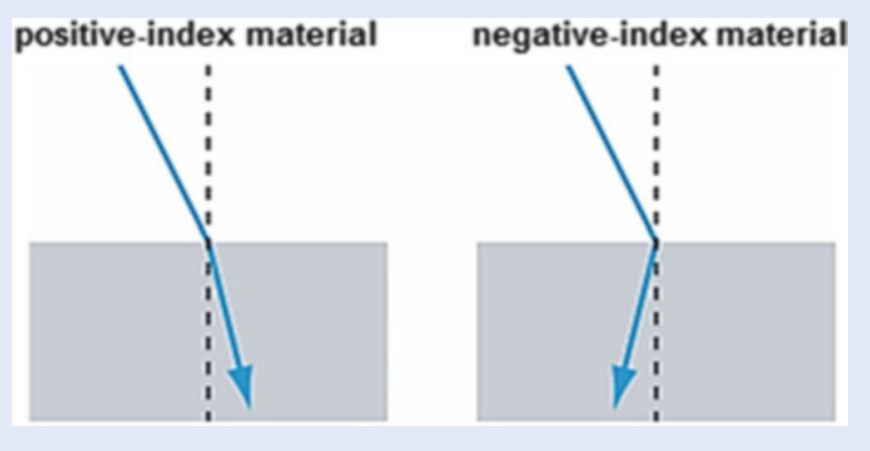

Fig. 10.10 Schematic of light refraction at a plane interface of dielectric-dielectric interface and at a dielectric - metamaterial (negative index) interface

\subsubsection{Heat Loss: Are Plasmonic-Based Devices Practical?}

One pivotal problem inherit in plasmonic technology is heat loss. Because plasmonic devices involve light interaction with metal, it necessarily involves energy loss due to heat dissipation. Metals are plagued by large losses due to strong electronic interband absorption, especially in the visible and UV spectral ranges. Among all metals, noble metals of gold and silver show the least energy loss. That is why gold and silver are used in most common plasmonics. Even in gold and silver, losses in the optical range including near- and mid-infrared (IR) regions are effectively still too high to make practical plasmon-based devices. Therefore, the search is continuing to find different approaches, such as creation of alloys and composites to make plasmonic materials that exhibit lower losses [22].

\subsection{Optics in Nanosemiconductors}

The interaction of semiconductor nanocrystals with light is fundamentally different from their bulk counterpart. Quantum dots, which were first discovered in 1980, are tiny particles or nanocrystals of a semiconducting material with diameters in the range of $2-10 \mathrm{~nm}$. In this size regime, additional quantum effects start to play an important role that can significantly alter some properties of the original material, such as optical activity. The most apparent result of this is that nanocrystals can fluoresce in distinctive colors determined by the size of the particles.

\subsubsection{Bandgap and Excitons}

Unlike metals, semiconductors do not electrically conduct, i.e., have no free electrons in the conduction band. All of the electrons are bound to atoms, i.e., they are in the valence electronic bands. This is because in metals the conduction and valence bands overlap but in semiconductors the edges of bands are separated by an energy gap called bandgap, as shown in - Fig. 10.11a [23].

In semiconductors an external agent such as external light is needed to impart enough energy to a bound electron to overcome the bandgap energy, as shown in - Fig. 10.11b. When this takes place the electron is placed in the conduction band accompanied with its separation from the positive charge. Separation of the electron from the positive charge leaves behind in the valence band what is called 


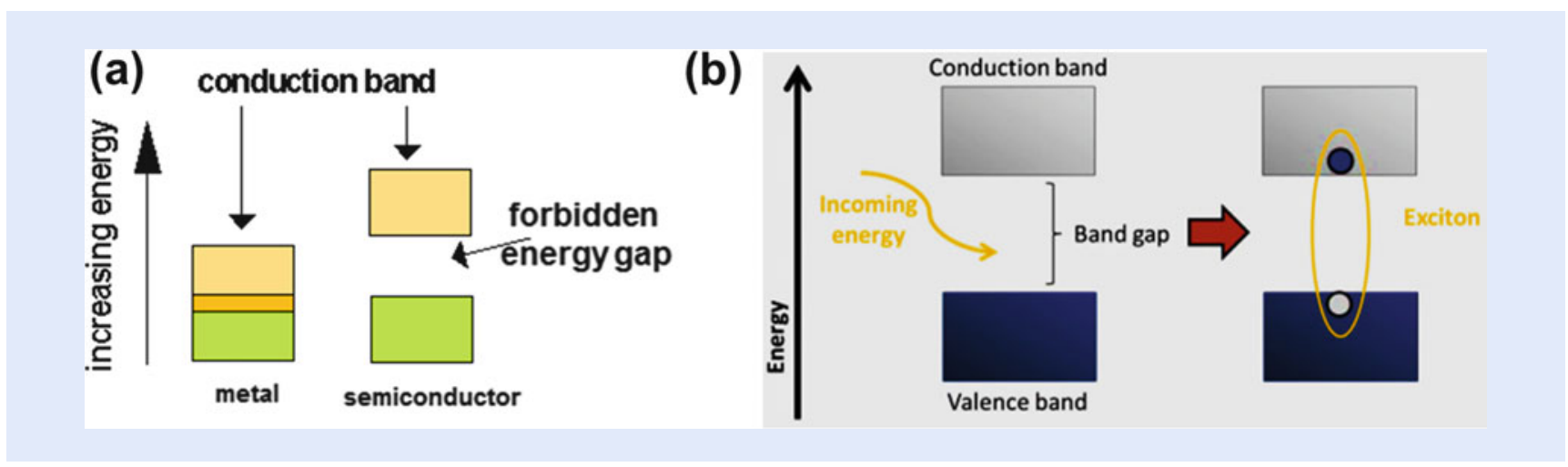

Fig. 10.11 (a) Conduction and valence bands for metal and semiconductor (Adapted from $>$ http://webs.mn.catholic.edu.au/physics/emery/ hsc_ideas_implementation.htm). (b) Light excitation of semiconductor elevating an electron from the valence band to the conduction band creating an exciton (Adapted from [23])

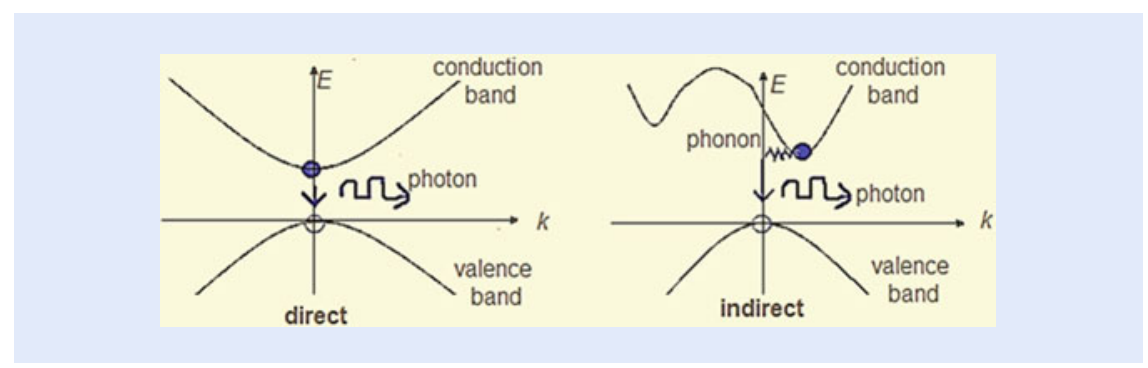

- Fig. 10.12 Schematic of the band structure showing the conduction and valence bands for direct bandgap material and indirect bandgap material

a positive hole. The electron as well as the positive hole are free to move away from their original common site; but they move together as an electron-hole pair or exciton, with the electron orbiting around the hole at an average distance or exciton Bohr radius. In silicon, for example, the Bohr radius is $4.2 \mathrm{~nm}$. The exciton structure is actually an "atom" which in some respect resembles the structure of a hydrogen atom, but 100-200-fold larger. Because of the large size, excitons are less bound and more fragile.

\subsubsection{Direct and Indirect Bandgap Materials}

The interaction (absorption or emission) of light with matter must satisfy the conservation of energy and momentum. The ability of meeting these conservations laws depends on the type of material. Two classes of semiconductors are interesting in this regard: indirect bandgap materials such as silicon and direct bandgap semiconductor such as CdSe (see • Fig. 10.12). The bandgap is called "direct" if the momentum of electrons and holes is the same in both the conduction band and the valence band. In this case an electron can directly emit a photon, which conserves energy while the momentum is automatically conserved, as shown in - Fig. 10.12 (left). In an "indirect" gap material, the momentum of electron and hole are not the same; hence a photon cannot be readily emitted because, to conserve momentum, the electron must pass through an intermediate state and transfer momentum in the form of phonon quanta to the crystal lattice, as shown in - Fig. 10.12 (right). The probability of coincidence of three particles: electron, 
hole, and phonon with specific properties is significantly lower than the coincidence of only two such particles (electron and hole). Thus, the probability of emission of a photon is much lower in indirect bandgap semiconductors than in direct bandgap ones. Bulk silicon is therefore a very poor light emitter, while in most cases, the direct bandgap semiconductors are good light emitters. Direct bandgap material is therefore used in light emitting devices, while indirect bandgap material is used in electronic devices.

\subsubsection{Enhancing and Blue Shifting of Luminescence by Quantum Confinement}

As we shrink the size of direct semiconductor material, novel optical properties begin to emerge as we approach a certain characteristic size scale. The characteristic scale is the electron-hole distance (the Bohr radius) of the material. For example, for CdSe the Bohr radius is $5.6 \mathrm{~nm}$. In this regime, the interaction of light with the material gets modified because quantum quantization of the energy levels of the electron as well as that of the hole according to Pauli's exclusion principle becomes important. A simplified treatment considers the energy of the electron and the hole as the energy of the charge in an infinite well (see - Fig. 10.13). The top well is for an electron and the bottom inverted well is for a hole.

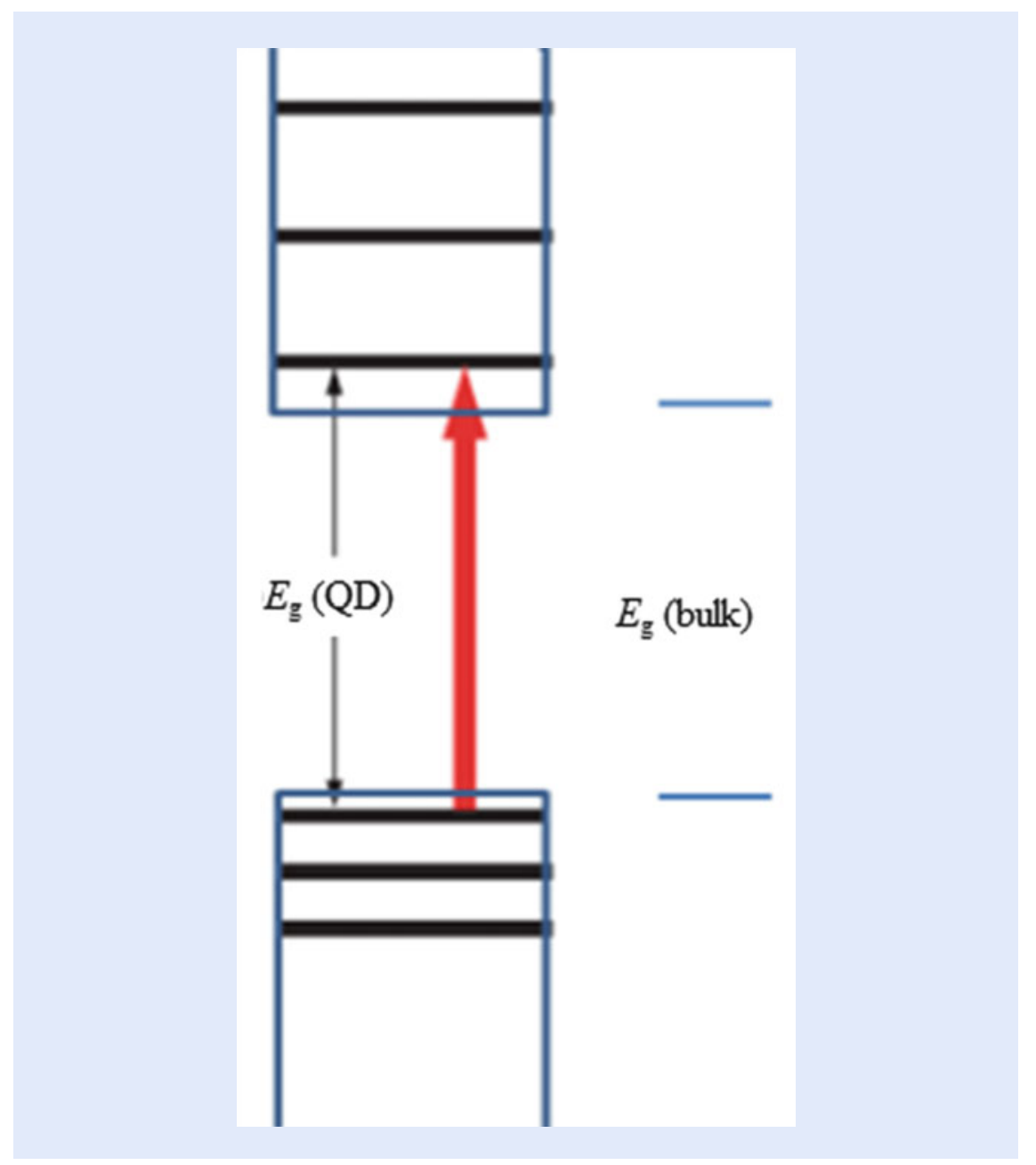

- Fig. 10.13 Simplified infinite well model of the energy of the electron and the hole in a quantum dot. The top well is for an electron and the bottom inverted well is for a hole 


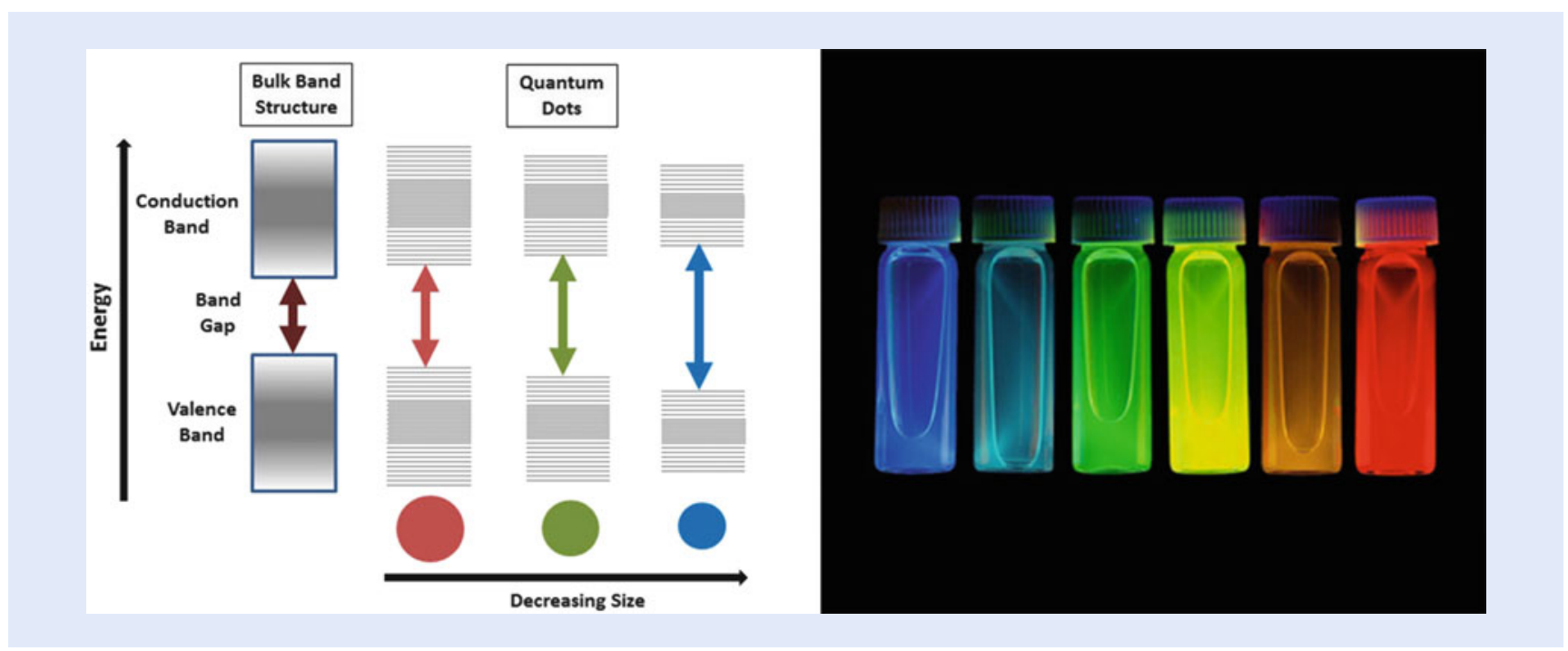

Fig. 10.14 (Left) The conduction and valence bands with decreasing size of a semiconductor nanoparticle (quantum dot), showing splitting of energy levels due to the quantum confinement effect. The bandgap increases with decrease in size of the nanocrystal (Images from ( $\downarrow$ http://www. sigmaaldrich.com/materials-science/nanomaterials/quantum-dots.html)). (Right) Vials of quantum dot colloids of increasing average size from left to right emitting light with color from blue to red respectively (Image from $>$ http://nanocluster.mit.edu/research.php [24])

This model results in three effects. First the energy levels become discrete according to: $E_{n}=\left(h^{2} n^{2}\right) /\left(8 m_{\mathrm{c}} R^{2}\right)$ where $n$ is an integer designating energy levels, $h$ is Planck's constant, $m_{\mathrm{c}}$ is the effective mass of the electron and hole system, and $\mathrm{R}$ is the radius of the quantum dot (particle). In this simplified model, the bandgap of the material widens to $E_{\mathrm{g}}=E_{\mathrm{g} 0}+\left(h^{2}\right) /\left(8 m_{\mathrm{c}} R^{2}\right)$. Second, this result shows that as the particle decreases in size, the bandgap energy increases, as shown in - Fig. 10.14 (left). More energy is then needed to excite the dot, and concurrently, more energy is released when the crystal returns to its ground state, resulting in a color shift towards blue in the emitted light. Third, in addition to the shift towards the blue the emission becomes stronger due to the discrete nature of the levels, making the nanoparticles much brighter than bulk [25]. As a result of this phenomenon, quantum dots can emit any color of light from the same material simply by changing the dot size. Additionally, with control over the size of the nanocrystals, quantum dots can be tuned during manufacturing to emit any color of light, as shown in • Fig. 10.14 (right).

\subsubsection{Making Silicon Glow: Quantum Confinement}

Silicon is the eighth most common element in the universe by mass, but very rarely occurs as the pure free element in nature. Monocrystalline silicon, manufactured from sand using sophisticated technology, is the backbone of the microelectronics industry. However silicon is the dullest material with regard to its optical activity because it belongs to the class of indirect bandgap semiconductors. - Figure 10.15 shows the energy-momentum diagram for silicon. Light emission in silicon is highly improbable because it requires both conservation of energy, which is satisfied by the emission of the appropriate photon energy at $1.1 \mathrm{eV}$, and conservation of momentum, which is satisfied by emission of a specific phonon (vibration of the crystal) of $55 \mathrm{meV}$ energy. Processes that require simultaneous emission of light and vibrations are highly unlikely and take a long time to happen, providing emission lifetimes on the order of milliseconds. In direct 


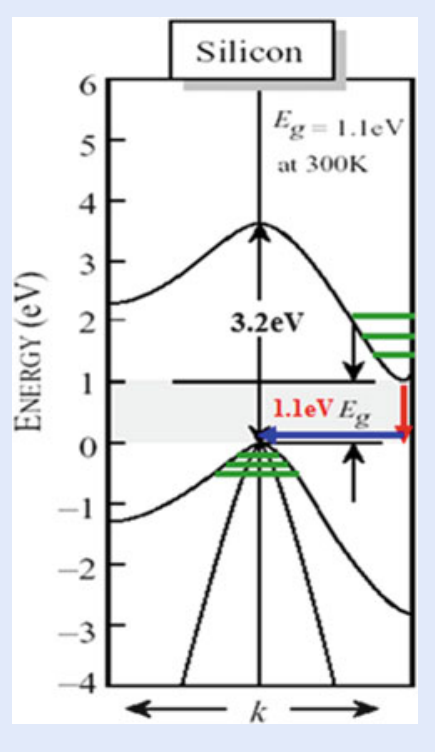

- Fig. 10.15 The energy-momentum diagram of the band structure of silicon. Emission of light requires both conservation of energy, which is satisfied by the emission of the appropriate photon energy at $1.1 \mathrm{eV}$ (red vertical arrow), and conservation of momentum, which is satisfied by emission of a specific phonon (vibration of the crystal) of $55 \mathrm{meV}$ energy (horizontal blue arrow). The upper set of green lines are discrete levels due to confinement of an electron and the lower set of green lines are due to the confinement of a hole

semiconductors, light emission proceeds readily with a lifetime of nanoseconds as the momentum is automatically satisfied without vibration and emission of phonons.

Recent developments enabled by nanotechnology are starting to change the picture. Some silicon nanostructures provide interactions with light now approach or even exceed the performance of equivalent direct bandgap materials, which promise to take silicon into the realm of optics. A significant deviation from bulk properties was found in 1990 when L. T. Canham noticed visible photoluminescence in porous silicon he produced via chemical etching of bulk silicon in HF acid [26].

But porous silicon is just an interconnected nanoscale network of silicon skeletal (sponge-like structure). The first report of micro- and nanoparticles prepared from porous $\mathrm{Si}$ came from the Sailor group at University of California in San Diego [27]. Strong ultrasound was used to shatter porous silicon into microand nanoparticles. But the particles do not have specific configuration or uniformity because silicon is a hard material and the particles are basically a result of mechanical stress and shattering of the interconnected skeletal backbone. In 1997, a new self-limiting etching procedure was developed by the Nayfeh's group at the University of Illinois which produced on the silicon wafer disconnected individual spherical nanoparticles of preferred or magic configuration or sizes, which can be softly retrieved and stored in a liquid of choice [28-32]. The particles are protected by mono-hydride coating and can be produced in commercial amounts and stored for later use for many years. The smallest of these particles is $1 \mathrm{~nm}$ and fluoresces in the blue. Other sizes include $1.7 \mathrm{~nm}$, fluorescing in the green; $2.15 \mathrm{~nm}$ fluorescing in the yellow-orange; and $2.9 \mathrm{~nm}$ fluorescing in the orange-red. - Figure 10.16 (left) shows the luminous of colloids of 1 and $2.9 \mathrm{~nm}$ particles in alcohol using excitation at a wavelength of $365 \mathrm{~nm}$. The 1-nm Si nanoclusters are amenable to testing and accurate first principle simulations because they consist of a manageable number of $\mathrm{Si}$ and $\mathrm{H}$ atoms and are produced in macroscopic 

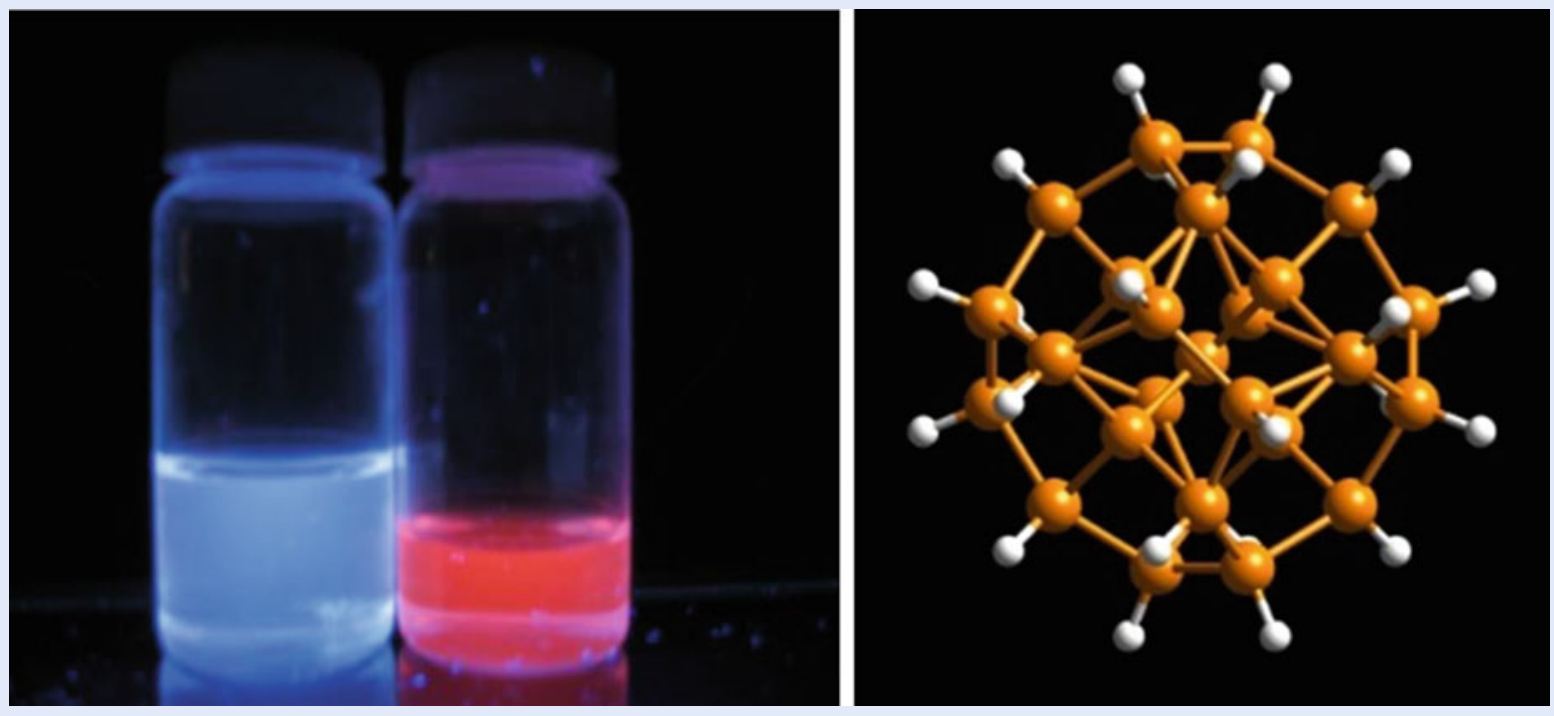

- Fig. 10.16 (Left) Blue and red luminescence of colloids of 1 and $2.9 \mathrm{~nm}$ particles in alcohol, respectively, with excitation at a wavelength of $365 \mathrm{~nm}$. (Right) A prototype structure of 1-nm particle has a configuration of $\mathrm{Si}_{29} \mathrm{H}_{24}$. Silicon atoms in orange. Hydrogen atoms in white

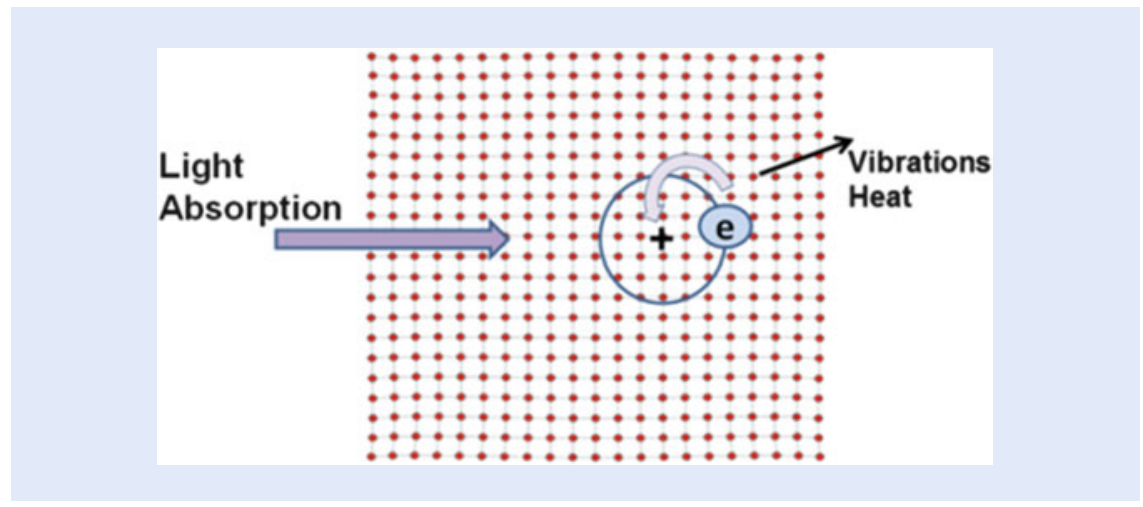

Fig. 10.17 Response of bulk silicon to light. Exciton forms followed by recombination to produce vibrations and heat before it can emit a photon

amounts. Those studies showed that the 1-nm particle simulated by Lubos Mitas has a configuration of a super molecule $\mathrm{Si}_{29} \mathrm{H}_{24}$, as shown in $\bullet$ Fig. 10.16 (right) [33-37].

One important characteristic of these particles is that the energy and momentum conservation rules governing light interaction get modified. In bulk silicon crystals or large particles, photo produced excitons move freely in all directions. Because it is not easy to conserve the momentum in indirect gap material when light is emitted, they recombine again before light is emitted with energy turning into vibrations and heat imparted to the crystal, as sketched in • Fig. 10.17.

When the particle is reduced to a size smaller than the exciton radius, especially in the sub 3-nm regime, the particles become less rigid allowing the silicon atoms to move, relax, or adjust especially those on the surface. Moreover, excitons get more strongly confined spatially, causing the momentum of the electron and hole to spread out appreciably according to the Heisenberg uncertainty principle, such that their momentum distributions can overlap. In the overlapping region, the momentum conservation may be readily satisfied; thus recombination of the 

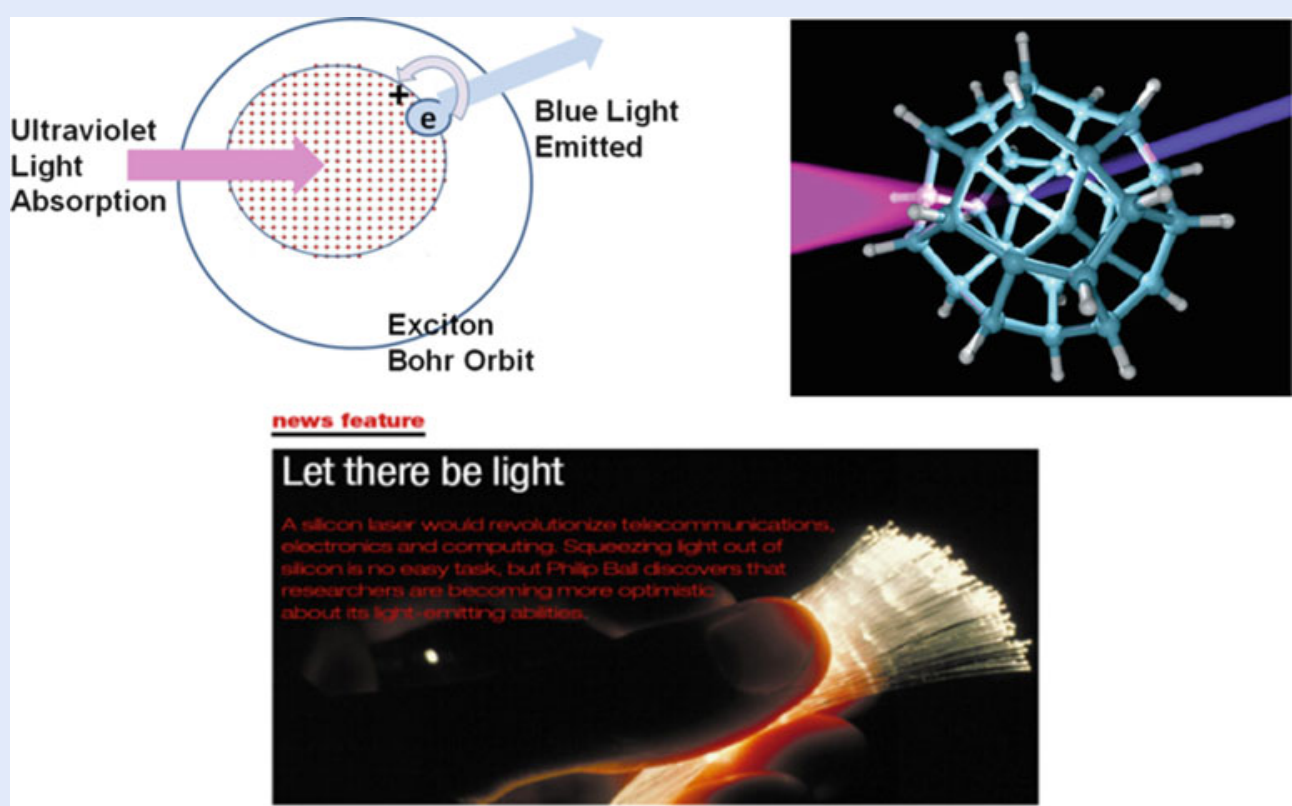

Fig. 10.18 (Top left) Schematic of response of 1-nm silicon nanoparticle to light. In 1-nm nanoparticle, much smaller than the Bohr radius, recombination produces a blue photon very fast much before vibrations and heat is produced. (Top right) Computer simulation image of 1-nm nanoparticle from [34]. (Bottom) Nature magazine report on the brightness of the silicon nanoparticles in terms of a biblical theme "Let there be light" (Image from [38])

electron and hole to turn the excess energy into light becomes stronger and faster than producing vibration and heat. In this case a photon (light) is produced, with the energy of the emitted photon (color of the light emitted) depending on the size of the particle.

For a nanoparticle size of $1 \mathrm{~nm}$, for example, emission of blue photons dominates the production of vibration and heat making it very bright with a performance that matches particles of direct bandgap material as in - Fig. 10.18 (top left) [39]. - Figure 10.18 (top right) is a computer simulation image of 1-nm nanoparticle carried out by researchers at Livermore National Laboratory [35]. Making silicon the optically dullest material in the universe glow is highly dramatic. Nature magazine reported this effect using the theme "Let there be light," as depicted in - Fig. 10.18 (bottom); it is a biblical theme of creation, signaling the transformation of the universe from the dark state to the bright state [38].

\subsubsection{Optical Nonlinearity in Nanosilicon}

The structure of a silicon crystal has cubic symmetry which exhibits inversion symmetry (centrosymmetry). But when the crystal becomes very small, it loses its rigidity and atoms especially the ones on the surface re-adjust, which exerts strain across the entire particle that alters the cubic structure and breaks the symmetry. Breaking the symmetry produces fundamental effects on its interaction with light. - Figure 10.19 (top left) displays one theoretical process that takes place in 1-nm particle in which some of the surface atoms lose their hydrogen atoms, move as much as $1.5 \AA$ each towards each other followed by reconstruction/connection to form $\mathrm{Si}$-Si dimer-like systems. 


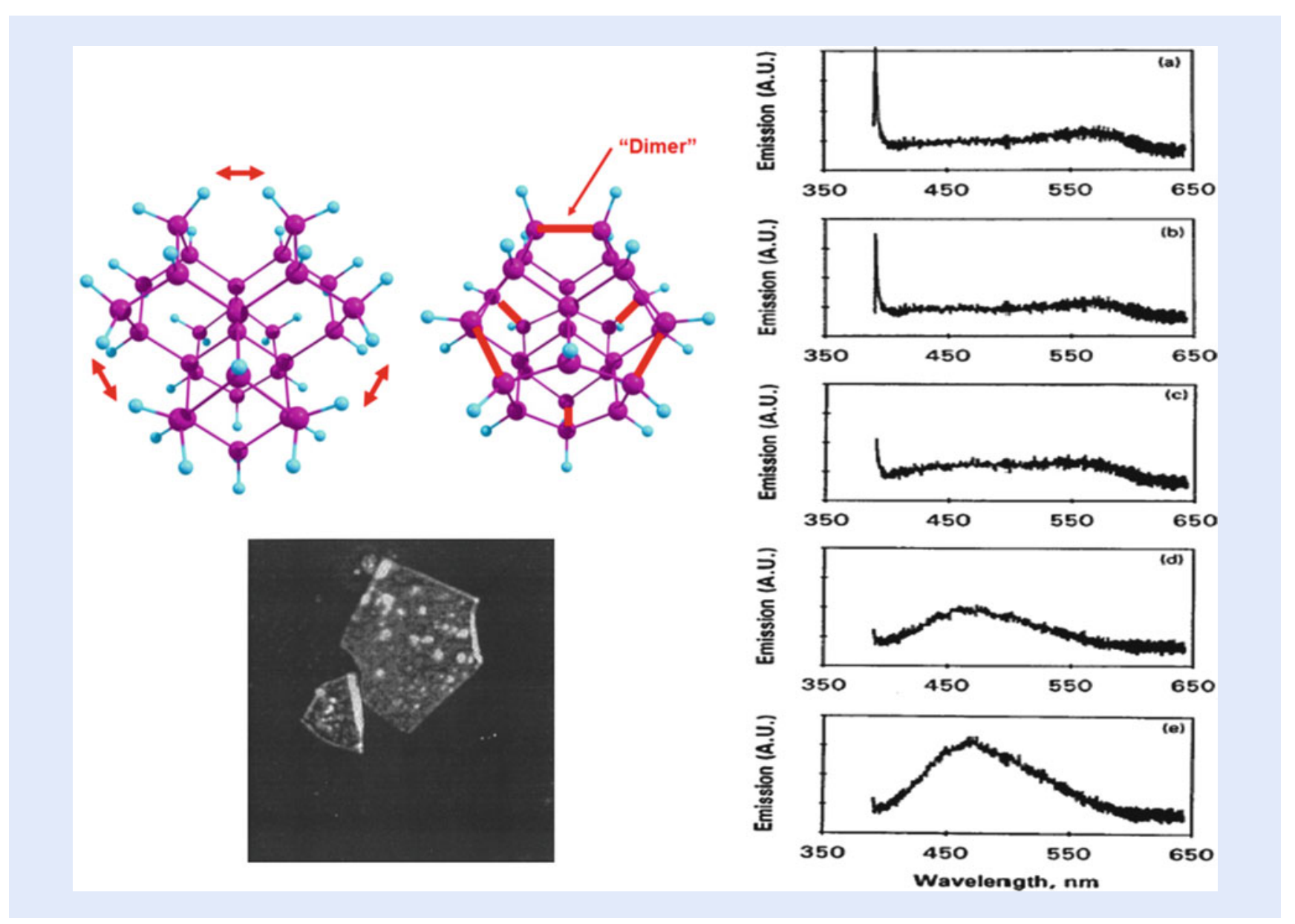

- Fig. 10.19 Response of microcrystallites of 1-nm silicon particles to 780-nm near-infrared femtosecond excitation. (Top left) Model of surface reconstruction that breaks the crystal symmetry of silicon. (Left bottom) Image of reconstituted microcrystals. (Right) Shows strong blue luminescent band indicating two-photon excitation as well as competing sharp radiation at half the wavelength of the incident beam. The intensity of the second harmonic anti correlates with the wide band luminescence

Blue luminescent 1-nm nanoparticles were used to test this mechanism. Particles prepared in water solvent were dried on device-quality silicon, which allowed them to form microcrystallites films, as shown in - Fig. 10.19 (left bottom). The microcrystallites were then excited by high intensity near-infrared femtosecond laser at 800-nm wavelength. • Figure 10.19 (right) displays several frames of the emission from different crystallites. It shows strong wide blue luminescent band as well as competing sharp radiation at half the wavelength of the incident beam (second harmonic). The intensity of the second harmonic anticorrelates with the wide band luminescence as seen in the figure. Both the sharp and wide band response indicates very strong coupling with light and the emergence of second-order nonlinear phenomena. The excitation of the blue luminescence band indicates a two photon excitation process, while the second harmonic indicates the breaking of the centrosymmetry. Other theoretical simulations showed that the mechanical strain exerted is extremely high, corresponding to a pressure of several Gpa [40]. Because atoms on the surface are under high strain, they can undergo large-amplitude molecular-like vibrations to relieve the strain [41]; and hence can also couple efficiently to thermal and mechanical stimuli. Second-order nonlinearity in silicon opens up other prospective applications in optics, including modulation, amplification, gain and laser action, and signal processing [42]. 


\subsubsection{Optical Gain in Nanosilicon-Based Material}

Not only miniaturization afforded silicon strong luminescence and optical nonlinearity, but there are also some preliminary reports indicating optical gain and laser action in films of silicon nanoparticles [43]. In one report [44], silicon nanoparticles were created inside a glass slab by silicon ion implantation followed by high temperature annealing that allows the silicon atoms to condense into silicon nanoparticles of $\sim 3-\mathrm{nm}$ diameter. The slab acted as an optical amplifier of weak light beams. In other reports 1-nm silicon nanoparticles were reconstituted into microcrystallites [45]. The crystallites were irradiated by infrared femtosecond pulses of a laser beam with high peak power. Microscopic directed blue emission was observed. Microscopic red emission was also observed from clusters of 2.9-nm nanoparticles under excitation of strong incoherent green light [46].

\subsection{Applications of Optics in Nanotechnology}

Miniaturization has triggered strong light-matter interactions. Nanotechnology allowed researchers to study light-matter interactions at the nanoscale and to launch the subfield of nano-optics. A considerable amount of basic knowledge as well as novel functions of nanomatter has accumulated over the past 20 years. Some applications have already reached the practical level, while others are futuristic. In this section we briefly present some practical applications of optics in nanotechnology in service of fields as diverse as electronics, opto- and photoelectronics, elementary particles, biomedicine, energy harvest and lighting, and art as in stained glass and lusterware pottery.

\subsubsection{Integration of Optics and Electronics}

Bulk semiconductors, especially silicon, form the backbone of modern electronics and computing. Bulk silicon, however, is a very dull material, being an especially poor emitter of light, turning added energy into heat. This makes integrating electronic and photonic circuits a challenge. There have been several proposals to alleviate this problem. One approach involves doping silicon with other materials. However, the emitted light is not in the visible rather in deep infrared. Moreover, the emission is not very efficient and can degrade the electronic properties of silicon. Another approach is to use nanotechnology by making silicon devices that are very small, such as using luminescent nanoparticles, five nanometers in diameter or less as introduced above. As we have seen above, at that size quantum confinement effects allow the device to emit light. But making electrical connections at that scale is not currently feasible and may compromise the optical activity of the nanomaterial [47], as well as afford very low electrical conductivity.

Another scheme involves subjecting bulk silicon to concentrated fields of plasmonics. The high fields afforded by plasmonic nanometal material can cause distortion and modification of the crystal structure, hence the interaction with light [48-50]. We describe briefly an architecture that demonstrates some of these effects. First a pure silicon nanowire is wrapped with a coating of glass, as shown in - Fig. 10.20 (left). Then it is mounted on a glass substrate. Then it is coated with silver. Because of the glass substrate, silver does not wrap completely around the wire, effectively making the silver coating take the shape $\Omega$. This leaves a narrow transparent glass window through which a laser beam can be sent in and emitted light can be extracted [48]. Practically, this narrow window did not compromise 


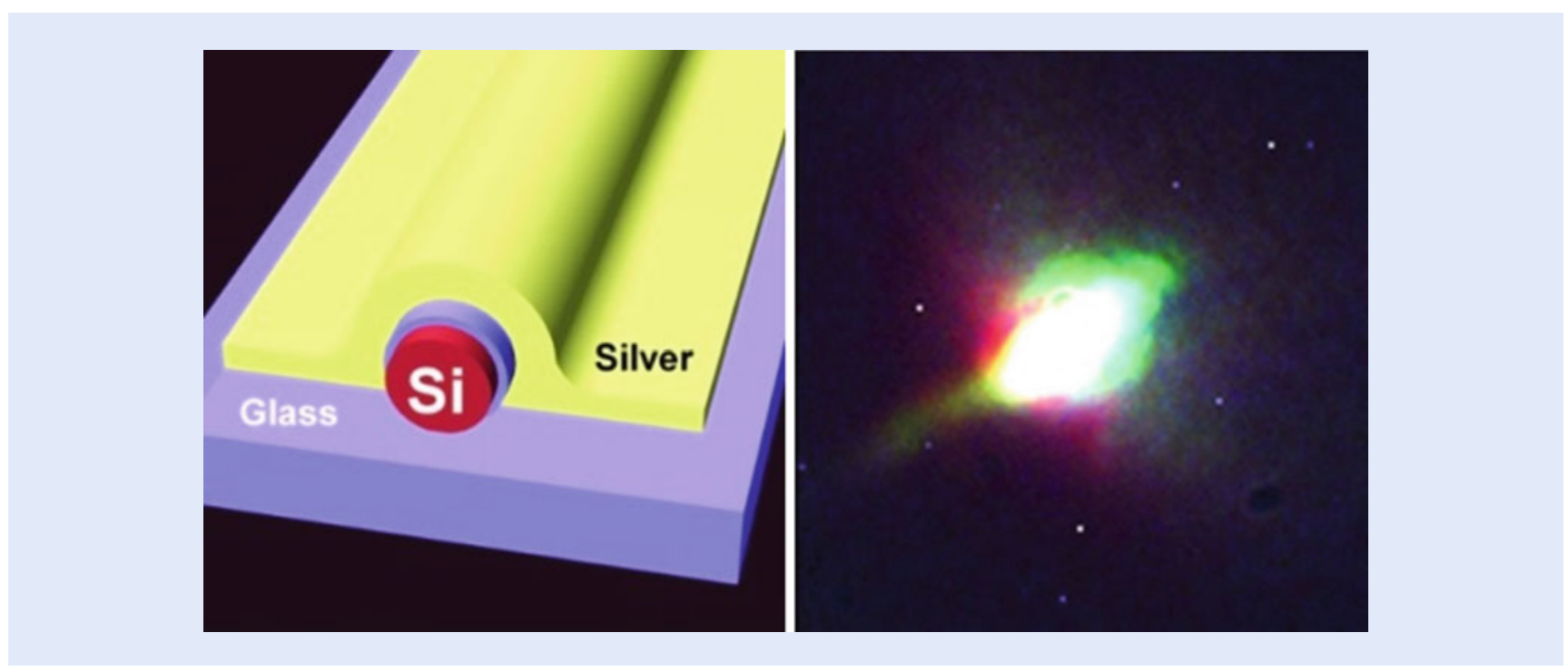

Fig. 10.20 Schematic of a hybrid silicon-silver nanowire system. (Left) Silicon nanowire wrapped with a glass coating followed by silver coating in the shape of $\Omega$, on a glass substrate. (Right) white light emission when a laser beam irradiates the system through the $\Omega$ window. (From [48])

the silver coating from acting as a plasmonic cavity. When a narrow band blue laser beam is sent through the window, the silicon wire produces white light that spans the visible spectrum, as shown in - Fig. 10.20 (right). It is to be noted that bare silicon wires do not produce such white light when excited by the blue laser, indicating plasmonic effects. The broad bandwidth light provides good potential for operation in photonic or optoelectronic devices.

\subsubsection{Confined Light in Service of Substance Detection}

Understanding interactions between strong light and matter is central to many fields. Deeper understanding of strong light coupling with matter is expected to afford the creation of advanced tailored applications [50]. The designation "strong field" applies to an external electromagnetic field that is sufficiently strong to cause significant alterations in atomic or molecular structure and dynamics of the material. Present-day laser technology as well as light enhancement due to plasmonic effects has made it possible to study the behavior of atoms and molecules in fields that have peak electric field strength of the order of atomic fields inside atoms or molecules. Under these conditions, even tightly bound ground states must be greatly altered by the presence of the field [51, 52].

Understanding interactions between light and nanomatter is central to many fields, providing invaluable insights into the nature of matter as well as the nature of light. Indeed, greater understanding of light-matter coupling has enabled creation of tailored applications, resulting in a variety of devices such as microscopic lasers, switches, sensors, modulators, fuel and solar cells, and detectors. As discussed above, hybrid-plasmonic monolithic nanowire optical cavities highlight recent progress made in tailoring light-matter coupling strengths.

Detailed Maxwell's theory was used to calculate the enhancement in intensity of light and the corresponding electric fields of light in the proximity of gold nanoparticles [53]. The results for 10-nm nanoparticles are given in • Fig. 10.21a, using a gray/white color code for incident light of $1 \mathrm{~V} / \mathrm{m}$ electric field. The enhancement based on these theoretical estimations can be as large as a factor of 10 , which provides enhancements of two orders of magnitude in intensity. 
a

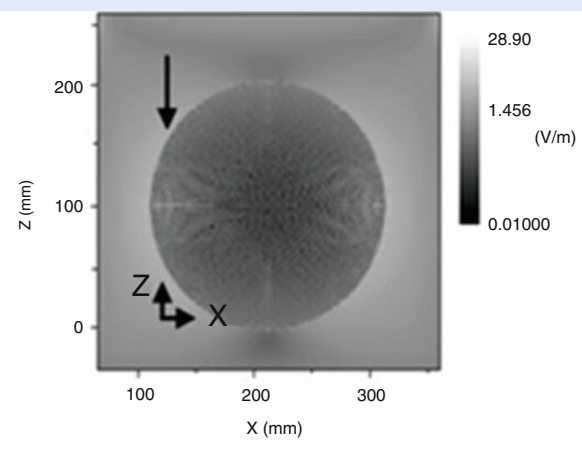

b

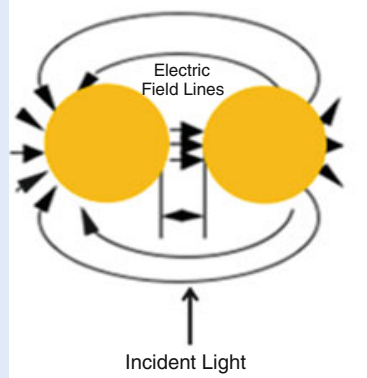

C

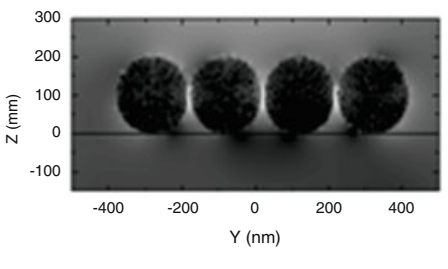

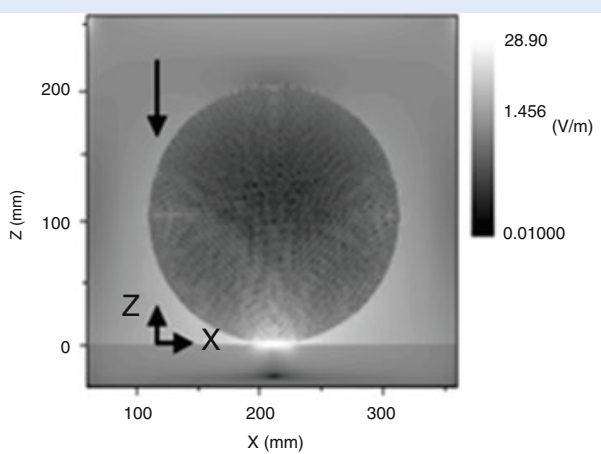

d

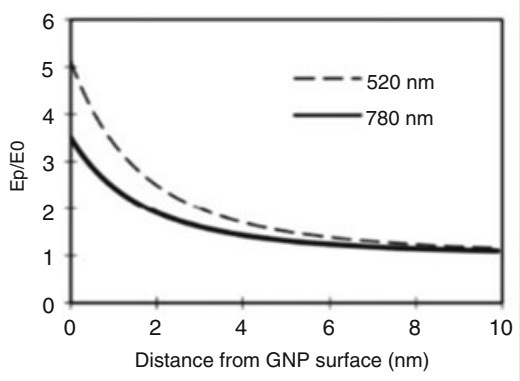

Fig. 10.21 Detailed Maxwell's theory was used to calculate the intensity of light and the corresponding electric fields of light in the proximity of 10-nm gold nanoparticles. (a) Near a single nanoparticles (b) schematic of the field lines between two particles as well as (c) actual results. Gray/ white color code is used for incident light of $1 \mathrm{~V} / \mathrm{m}$ electric field (d) detailed dependence, as an example, of the electric field near a gold nanoparticle illuminated with light of two different wavelengths. (From [53])

Enhancements can be even more dramatic when there is more than one metal particle. - Figure $10.21 \mathrm{~b}$, c displays a schematic of the field lines between two particles as well as actual results using the gray/white color code, respectively. It shows, in space between two closely placed plasmonic nanoparticles the electric field may be enhanced by nearly a factor of 30 , providing nearly three orders of magnitude enhancement in intensity. • Figure 10.21d gives detailed dependence on the wavelength of the light. As an example, the figure gives the electric field near a gold nanoparticle illuminated with light of two different wavelengths [53]

Thus the confined light to nanometal spaces is short range (dies out exponentially) and does not propagate to large distances. Because of the short range of the field, it interacts only with nanostructures next to it, within $\sim 10-30 \mathrm{~nm}$. Thus it provides spatial nanoresolution. This opens up many applications, especially those optical processes or phenomena that depend very sensitively on the magnitude of the electric field, such as fluorescence, Raman scattering, and infrared absorption, resulting in plasmon-enhanced fluorescence, surface-enhanced Raman scattering, and surface-enhanced infrared absorption spectroscopy.

The Raman scattering effect, for example, depends on the fourth power of the field; thus they are enhanced if scattering is performed with the confined light near a metal nanoparticle rather than with ordinary propagating light. The Raman scattering effect is used for sensing and identification of substances. When a substance is irradiated with light, it scatters light called Raman light at a wavelength or frequency slightly shifted from the wavelength of the original irradiated light by an amount equal to the natural frequency of molecules that make up the substance. So, detecting the Raman light and analyzing its spectrum allows the 


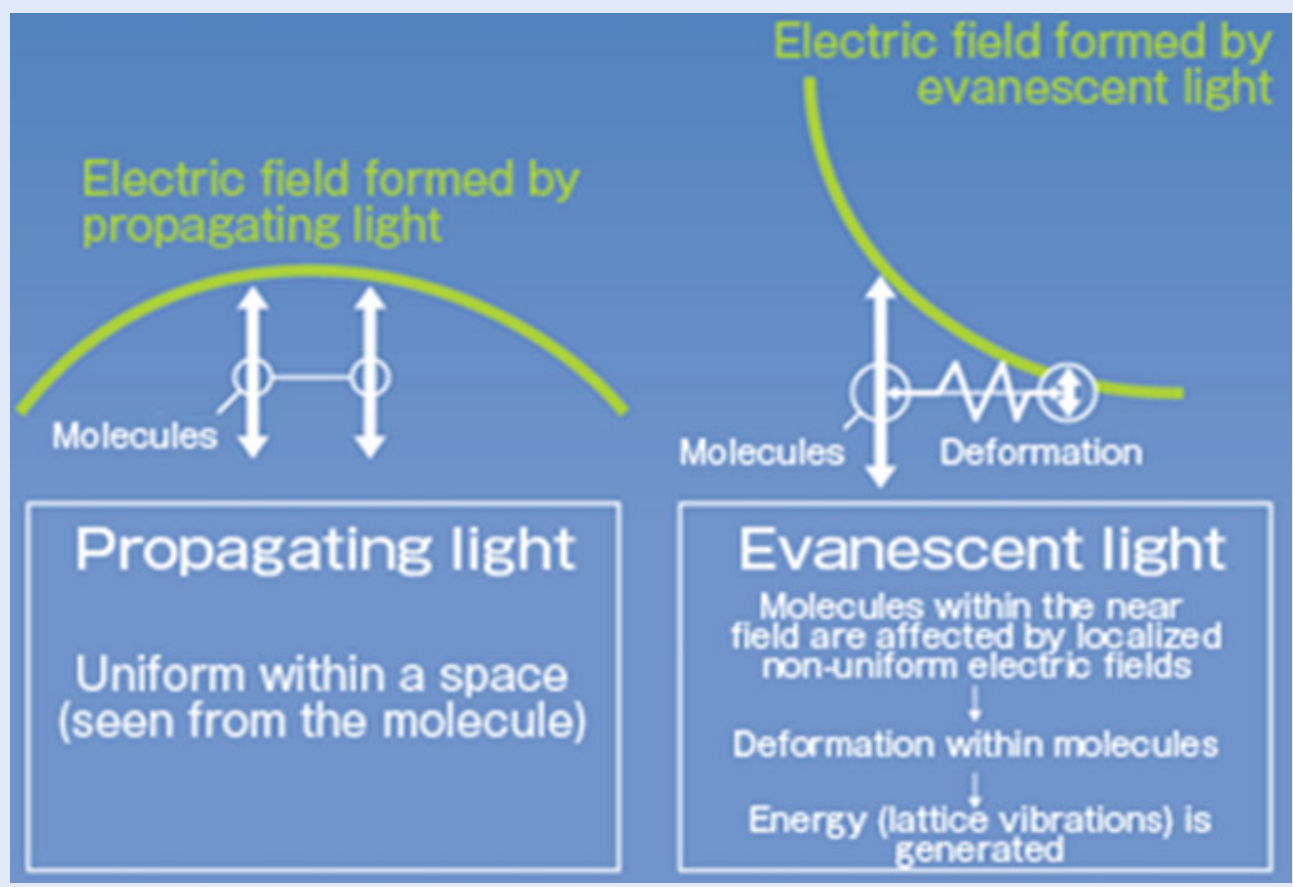

Fig. 10.22 Schematic of a large molecule in the proximity of metal nanoparticle. It shows the profile of the plasmonic electric field. Different parts of the molecule experience different electric field strengths, introducing strong distortion within the molecules (From [16])

identification of the substance. Since Raman light is usually very weak, detecting its intensity directly is quite difficult. If the electrical field strength is increased 10 times by surface plasmon resonance, then the Raman light is intensified $10^{4}$ times so the intensity becomes 10,000 times higher.

Another interesting phenomenon of strong fields is the ability to optically manipulate the structure of molecules. Since the electric field in the vicinity of the plasmonic nanoparticles drops very rapidly over $10 \mathrm{~nm}$, different parts of large molecules will experience different electric field strengths hence different electric stress. This causes distortion within the molecules and consequently causes new ways to deliver energy to the molecule including vibrations, nonlinearities, and electronic structure, as shown in - Fig. 10.22. The additional energy supplements the energy of the incident photon [16].

\subsubsection{Nanofabrication and Nanolithography}

Atoms or molecules are placed near the atomically sharp metal tip (tungsten or gold tip) of a scanning tunneling microscope (STM), as shown in • Fig. 10.23 (left top) [54,55]. Below the tip is a conducting substrate at a distance of $1 \mathrm{~nm}$. When the tip is biased by $1-3 \mathrm{~V}$, an electric field is setup in the gap which can reach more than $100 \mathrm{MV} / \mathrm{cm}$. An intense pulsed laser light bathes the metal tip and the gap and the substrate. The electric field in the vicinity of the gap is very strong due to the dc field of the tip as well as to plasmonic fields. The combined effect of the light and electric field is confined to a nanospace and will be very intense on atoms or molecules falling in this space near the tip. The intense pulsed laser light can be tuned to resonate with a certain state in an atom or molecule, promoting them to high-lying excited states while the combined electric field can assist in stripping the excited electron to produce a single ionization event of a free electron and a free ion. 


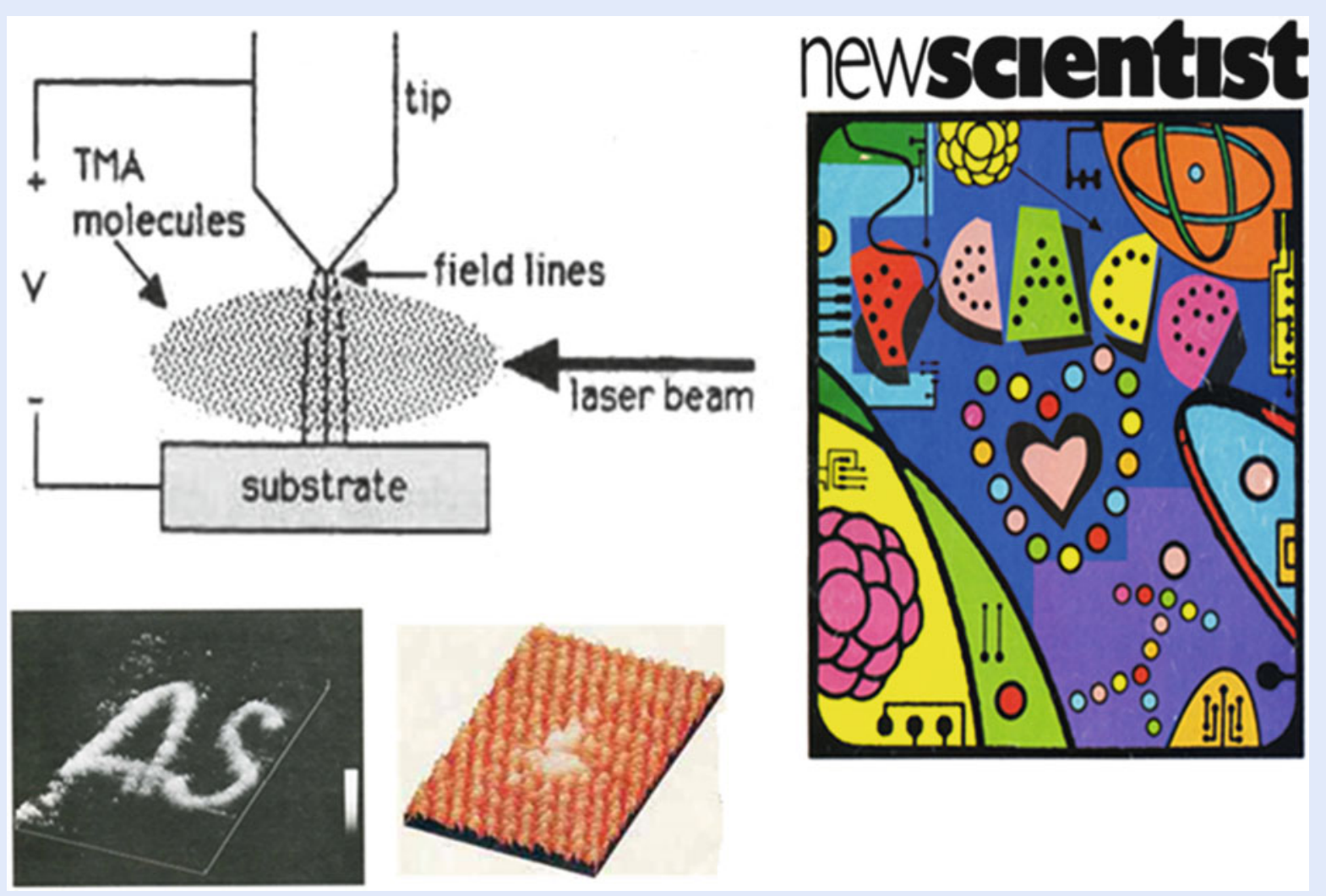

- Fig. 10.23 (Left top) Schematic of a tungsten tip of a scanning electron microscope bathed with a laser beam in a chamber with a certain pressure of a molecular gas. The metal tip is biased at 1-3 V. Molecules, in the gap or near the gap, are subjected to the combined effect of the laser field and the localized field of the metal tip excites, dissociates, ionizes, and pins down ions on the counter surface. (Left bottom) Magnified image of the initials of Arthur Schawlow "AS" written with trimethyl aluminum molecules on a silicon wafer. A single molecule of trimethyl aluminum is picked on a graphite surface. (Right) Cover of The New Scientist showing displaying a nanofabrication pattern in the form of a "heart" performed by the process depicted in the top left schematic (From [54]) along with patterns in the form of the "molecular man" by IBM and the word "PEACE" by Hitachi, both written using STM tips only

Since the electric field is focused or confined to nanoscale then the atomic ion is accelerated, pushed, and guided to the surface with nanoscale resolution. Programming the position of the tip allowed the Nayfeh's group to make nanopatterns on the surface with atomic resolution. Since light can resonate with specific atoms or molecules then the process is highly selective and can pick a certain atom from a mixture of atoms or molecules, as shown in $\bullet$ Fig. 10.23 (left bottom). In one of the earliest news media reports on nanotechnology under the title the "Smallest Graffiti in the World," and subtitle "Nanotechnology Rules," the British magazine, "new Scientist" in 1992 covered the process and displayed several patterns ("heart") on its cover along with the work from IBM ("molecular man"), and Hitachi of Japan ("peace") (• Fig. 10.23 (right)) as symbols of nanotechnology [54]. In 1992 on 70th birthday of Arthur Schawlow, the inventor of the laser the author presented him with the nanoscale of his initials " $A S$ " written with trimethyl aluminum molecules on a silicon wafer, shown in • Fig. 10.23 (left bottom). STM-based processing has been proposed by the community for nanolithography in the electronics industry, but since it relies on the movement of mass for patterning it was concluded that it is very slow and as such unpractical for mass production.

The electric field can also be confined in the vicinity of a very thin tungsten wire, such as that of a Geiger counter used in detecting and counting charged 


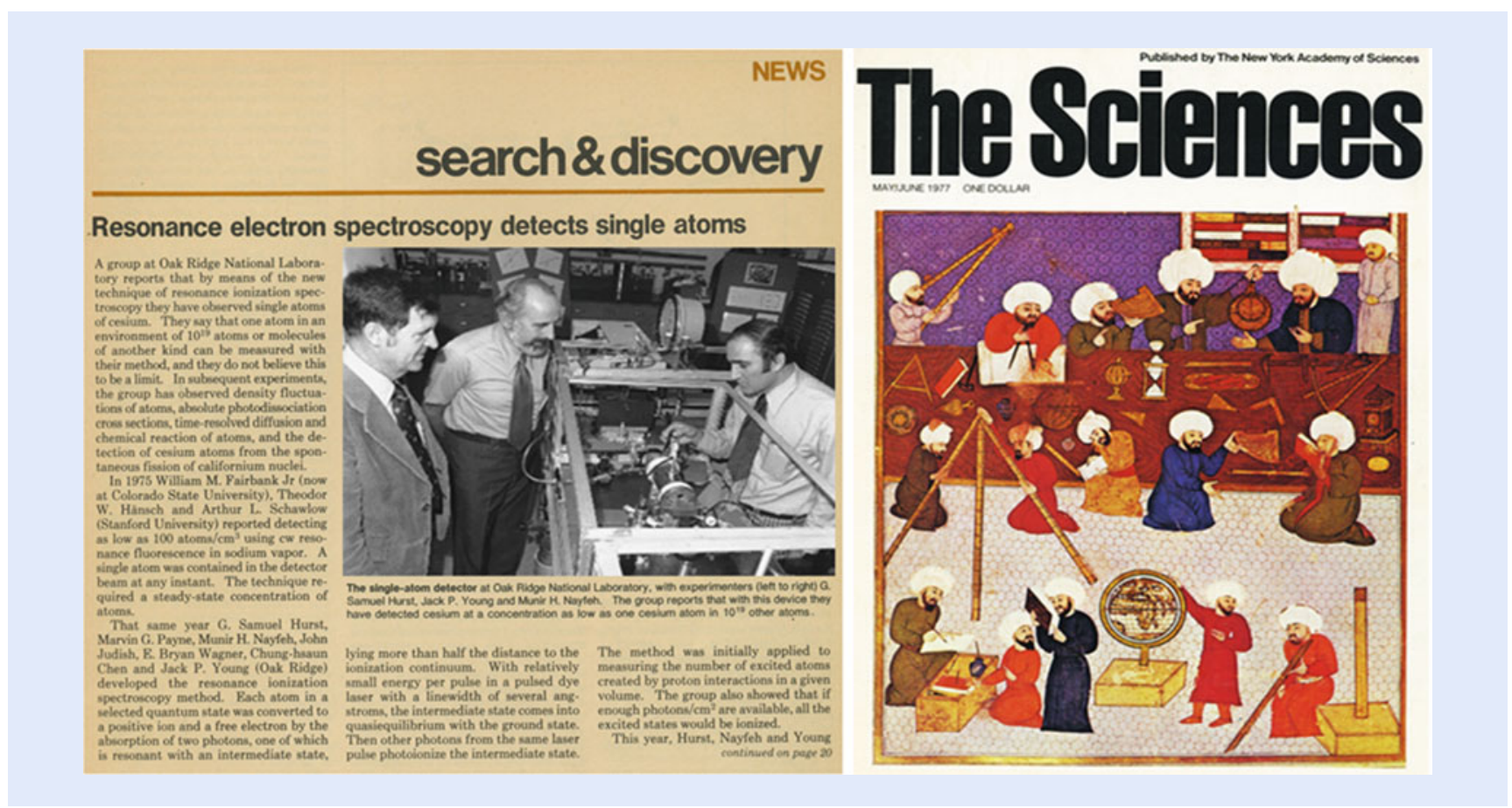

- Fig. 10.24 (Left) Report in search and discovery of Physics Today about the use of intense light combined with an electric field to identify and selectively detect single atoms (From [57]). (Right) cover of The Sciences displaying Alhazen conducting some laboratory experiments. Inside, the issue reports on the detection of single atoms using the process described in the Fig. 10.24 top left sketch (From [58])

particles for nuclear radiation research [56]. This configuration has been used in 1975-1977 for identifying and counting single atoms by Hurst, Nayfeh, and Young at Oak Ridge National Laboratory [57]. It is to be noted in 1975 William Fairbank Jr., Theodor Hansch, and Arthur Schawlow at Stanford University reported detecting as low as 100 atoms $/ \mathrm{cm}^{3}$ using $\mathrm{cw}$ resonance fluorescence in sodium vapor [57]. The Oak Ridge results were covered in the search and discovery news of Physics Today, as shown in - Fig. 10.24 (left) [57], and in The Sciences magazine published by the New York Academy of Sciences shown in - Fig. 10.24 (right) [58]. The cover of same issue of "The Sciences" shows a sixteenth century painting of Islamic astronomers at work in their observatory. One of the scientists shown is Alhasan Ibn Alhaytham (Alhazen). Because the process constitutes the ultimate sensitivity in analytic detection of matter, namely a single atom, the work was also covered in Britannica Yearbook of Science and the Future (1979), McGraw Hill Yearbook of Science and Technology (1979), The World Book Science Annual (1978), and more.

\subsubsection{Photovoltaics and Photocurrent}

One hot area involving the interaction of light with nanosemiconductor in the presence of an external electric field is the generation of voltage that can be stored. A thin film of silicon nanoparticles or capsules of silicon nanoparticles, for example, are placed on top of a silicon-based $\mathrm{p}-\mathrm{n}$ junction (amorphous, polycrystalline, or monocrystalline silicon solar cell). When light strikes the nanoparticles some light is absorbed. As a result, electron hole (e-h) pairs (excitons) are produced in the nanoparticles. If the electron and hole are separated from each other completely before they could recombine to produce light (luminescence) or 

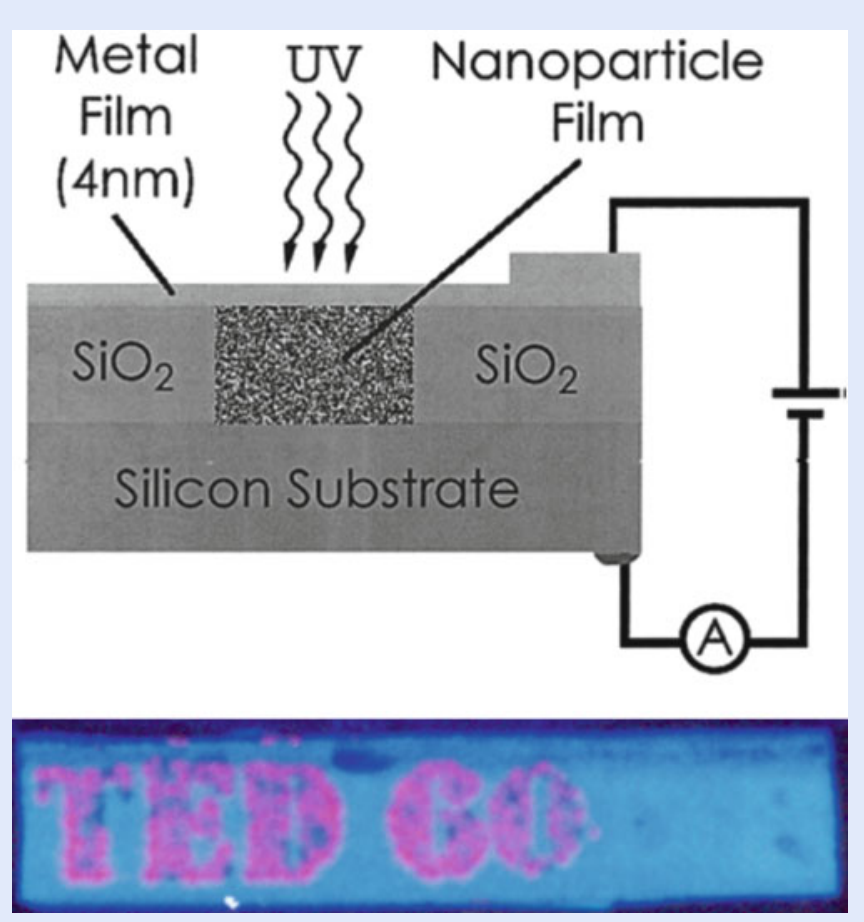

Fig. 10.25 (Top) Highly sensitive photodetectors for various applications consisting of holes filled with silicon nanoparticles and covered by an aluminum thin layer (bottom) highly magnified image of a pattern of holes in the shape of the words "TED 60" filled with red luminescent silicon nanoparticles under UV excitation was presented to Theodor "Ted" Hansch on his 60th birthday celebration in Munich [62]

recombine non-radiatively to produce vibrations and heat then the electrons and holes may be transported, collected, and stored on two external electrodes appropriately constructed and positioned. The voltage difference between the electrodes can be harnessed at a later time as a battery. The electric field in a p-n junction plays a pivotal role in charge separation and collection. This architecture using 2.9$\mathrm{nm}$ and 1-nm silicon nanoparticles showed relative power enhancements of the efficiency of the under lying solar cell in the UV and in the visible [59, 60, 61].

If the nanoparticles are placed simply on a simple conducting substrate instead of a p-n junction, then a continuous flow of electrons may proceed which may act as an instantaneous current source. A thin oxide layer is grown on a silicon wafer, followed by etching out a hole or multiple holes using a mask. The holes are filled with silicon nanoparticles and an aluminum thin layer is grown as a cap/electrode, as shown in - Fig. 10.25 (top). Architectures of this type have been used as highly sensitive photodetectors for various applications [62]. In one application, it provided a sensitive detector for UV radiation while being blind to visible radiation, property which is useful for elementary particle collisions in neutrino, dark matter, and rare decay experiments. In those weak UV Cherenkov radiation is produced that gives vital information about momentum and geometry of collision [63]. Following the same procedure using a mask in the shape of the words "TED 60 ", a red luminescent pattern was created, as shown in • Fig. 10.25 (bottom). In November 2001, the silicon wafer was presented to Theodor "Ted" Hansch on his 60th birthday celebration in Munich. Among the guests are four physics Nobel Laureates: Norman Ramsey, Steven Chu, Claude Cohen-Tannoudgi, and Carl Weiman. The image was published in 2005 in the IEEE Transactions on Nanotechnology just after Hansch received the Nobel Prize [62]. 


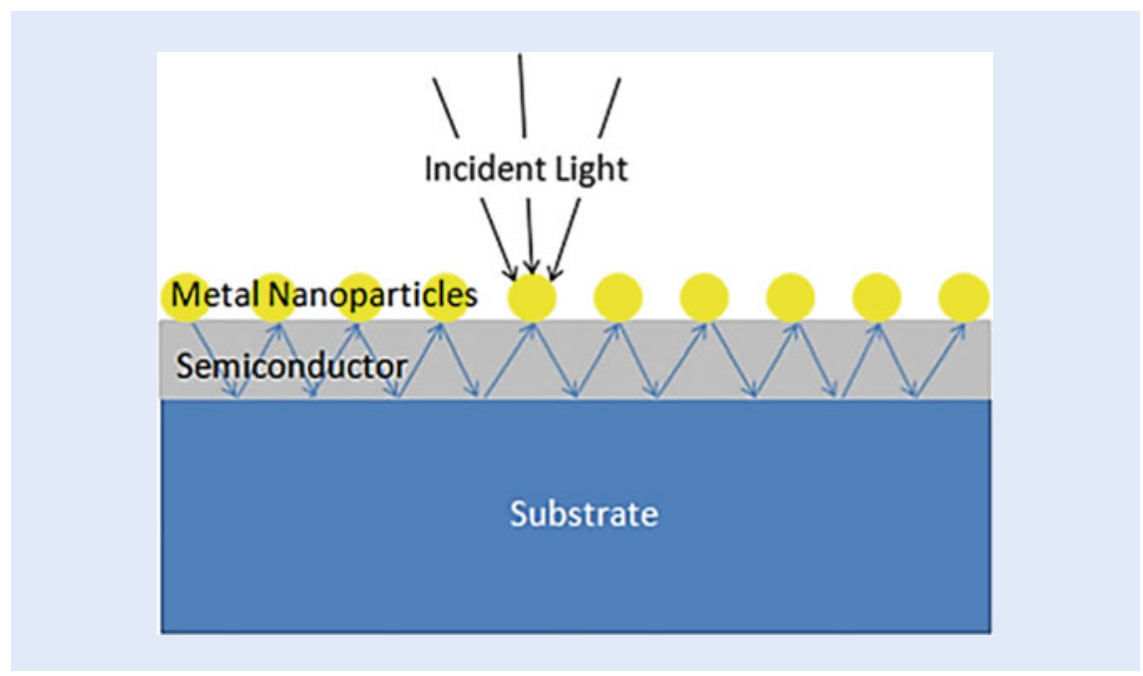

Fig. 10.26 Schematic of a plasmon solar cell. It consists of a thin silicon-based active layer on a glass substrate. Gold nanoparticles are placed on the active layer. (Adapted from $>$ https://en. wikipedia.org/wiki/Plasmonic_solar_cell)

In another development, silicon nanowire arrays were used [64]. Efficient procedures for fabrication of nanowires were recently developed [65, 66]. Hemispherical gold deposits are made on the end of the wires. When a near-infrared optical field falls onto the gold deposits, it excites plasmon resonances, which remarkably amplify the intensity, effectively making them like antennas. The same gold deposit can form a p-n like Schottky junction with the nanowire, which enhances charge collection. Thus the system acts as an effective near-infrared photodetector [64]. Silicon-based sensitive UV photodetectors have military as well as commercial applications. Military applications include missile warning systems, biological attack warning systems, and jet engine sensors.

Light interaction with nanometal is also emerging as a useful intermediate agent for improving light coupling to thin film solar cells; hence improving their efficiency [67]. Thin film solar cells utilize 1-2 $\mu \mathrm{m}$ thick semiconductor material placed over substrates of cheap material compared to silicon, such as glass, plastic, or steel. Metal nanoparticles are deposited on the top of the semiconductor, as shown in - Fig. 10.26. When light hits these metal nanoparticles at their surface plasmon resonance, light is scattered in many different directions. This allows light to travel along the active semiconductor and bounce between the substrate and the nanoparticles enabling the semiconductor to absorb more light even though it is a thin layer [67].

\subsubsection{Solid State LED White Lighting}

The objective of using electronic chips for lighting is to manufacture light bulbs that are superior to conventional bulbs yet with characteristics that match the sun's white light spectrum, given in - Fig. 10.27 (top). Light emitting diodes (LED) are chips powered by electricity. They produce light of specific color in the $\mathrm{UV}$, blue, green, or red light range. White light bulbs for domestic use can be manufactured by combing light from three LEDs: blue, green, and red. Because the emission of each LED is not wide enough, the mix produces "finger shaped" spectrum, not smooth as normal sun light (• Fig. 10.27 middle). The problem has been somewhat alleviated and simplified in recent years by using a single blue LED source and a wide band green/orange phosphor converter, which combines to 

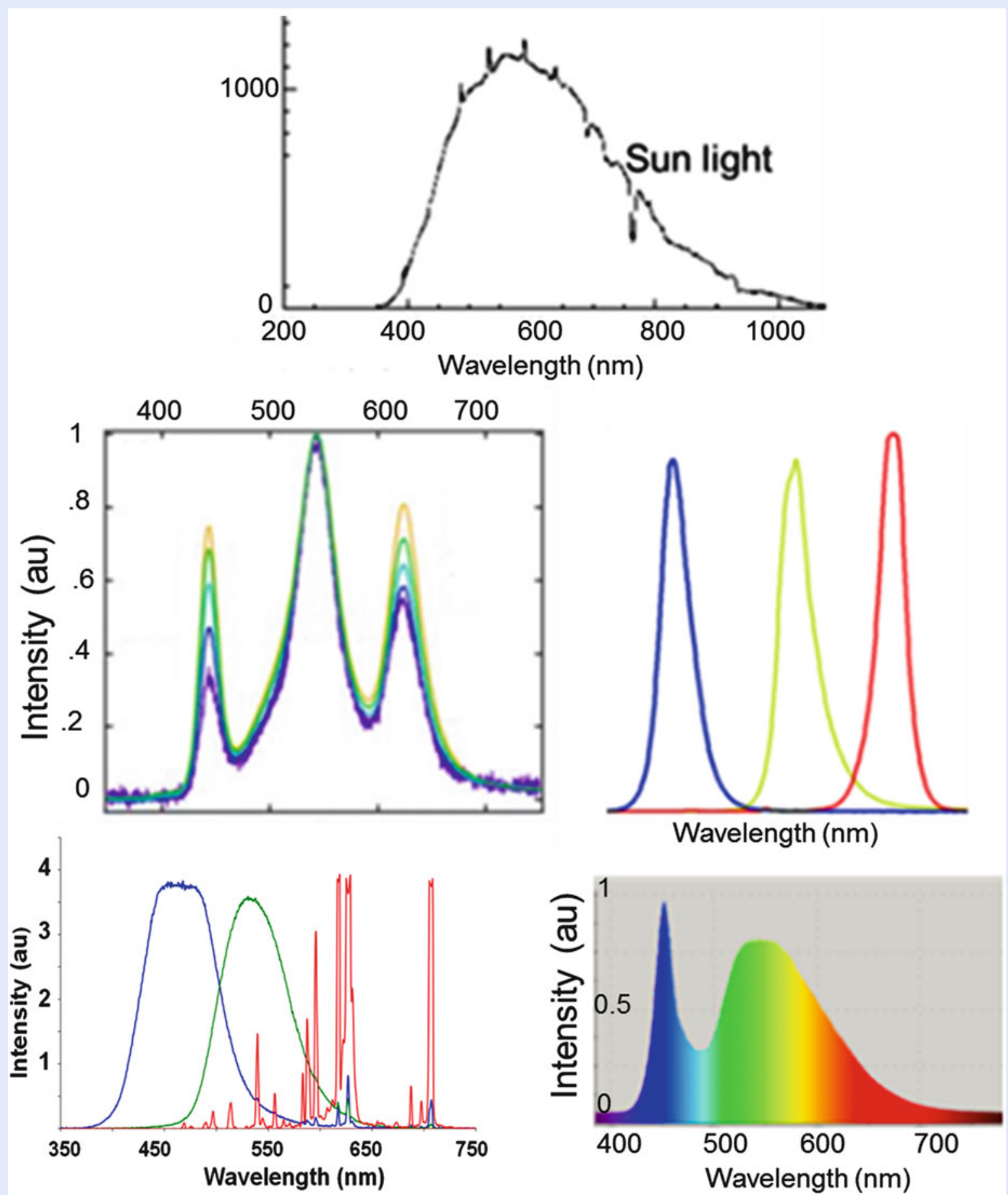

- Fig. 10.27 (Top) Spectrum of sun light (middle) mixing light from three LEDs: blue, green, and red, producing "finger shaped" spectrum not smooth as normal white sun light (bottom right) single blue LED source and a green/orange phosphor converter, which combines to a "hand shaped" spectrum not filled enough (bottom) single UV LED and three phosphors (blue, green, and red phosphor); red phosphor converters, based on Europium (Eu) provides spectra dominated by sharp red lines 
a better filled spectrum ( $\bullet$ Fig. 10.27 bottom right). Although the mix produces a "hand-shaped" spectrum and short of full match with sun light as it is missing a red component, the developers of this process Isamu Akasaki, Hiroshi Amano, and Shuji Nakamura were awarded the 2014 Nobel Prize in Physics. The first LED (red) was invented by Nick Holonyak at the University of Illinois several decades earlier ( $\triangleright$ http://www.led50years.illinois.edu/nicks-story.html), but it was deemed that the more recent blue LED (invented by the awardees) and its use in this configuration afforded a very important service, namely filling the world with new "white" light. A third solution is a UV LED and three wide band phosphor converters (blue, green, and red phosphor). However, there is a problem with red phosphor converters as they are based on europium $(\mathrm{Eu})$. In addition to problems of availability and stability, those provide spectra dominated by sharp red lines (• Fig. 10.27 bottom left), which produces a mixed spectrum with sharp lines not smooth enough as normal white sun light (• Fig. 10.27 top). In addition, phosphor films are found to have appreciable reflectivity causing a non-negligible fraction of the pumping LED light to be reflected backwards. This in turn causes heating and requires more power input. More energy efficient and rigorous designs must be used to minimize heating.

The novel optical properties of nanomaterial discussed above in $\bullet$ Sect. 10.3.3 may alleviate many of these problems. In fact, CdSe-ZnSe quantum dots or nanoparticles have been demonstrated as potential substitute for "red phosphor" for use in near UV pumping [68]. However, due to their direct nature, luminescence of a given nanoparticle size is sharply dependent on its size, which requires the use of an appropriate size distribution to produce broadened emission.

Silicon nanoparticles have also been demonstrated as substitute for or additive to Eu-based "red phosphor" [69], as shown in - Fig. 10.28. Because of the indirect nature of silicon, individual silicon nanoparticles produce inhomogeneous broadened luminescence (• Fig. 10.28 top), avoiding sharp-line mixing; and because of strong UV absorption they are versatile for pumping with the emerging powerful UV LEDs. It is hoped that UV-pumped "nano-phosphor" converters would allow manufacturing of high quality white bulbs that cover much larger areas while affording high color rendering index which is the quantitative measure of the ability of the bulb to reveal the colors of objects faithfully, while independently providing a correlated color temperature (CCT), which is a specification of the color appearance of the light emitted by the bulb relating its color to the color of light from a black body radiator heated to the same temperature. Commercials CCT labels include warm, daylight, and cool labels of temperature.

Another advantage of using nanomaterial as a component in the phosphor mixture stems from the fact that nanomaterial reduces the reflectivity of the mixed composite material so as not to harm the pumping LED chip. Also nanomaterial improves heat dissipation, thus prolonging the lifetime of the bulb. Thus current manufacturing process of white light can be developed further using the novel interaction of light with nanomatter to produce better efficiency bulbs that cover smoothly the solar white light range, as well as handle larger areas.

\subsubsection{Plasmonic Hyperthermic-Based Treatment and Monitoring of Acute Disease}

One serious problem inherit in plasmonics is energy loss due to heat generation resulting from strong absorption especially in the infrared-visible and UV spectral ranges [70, 71]. So far, the losses are effectively too high to make practical plasmon-based electronic or photonic devices. However, these losses turned out to be a blessing for other applications, especially for cancer therapy. In this treatment, 


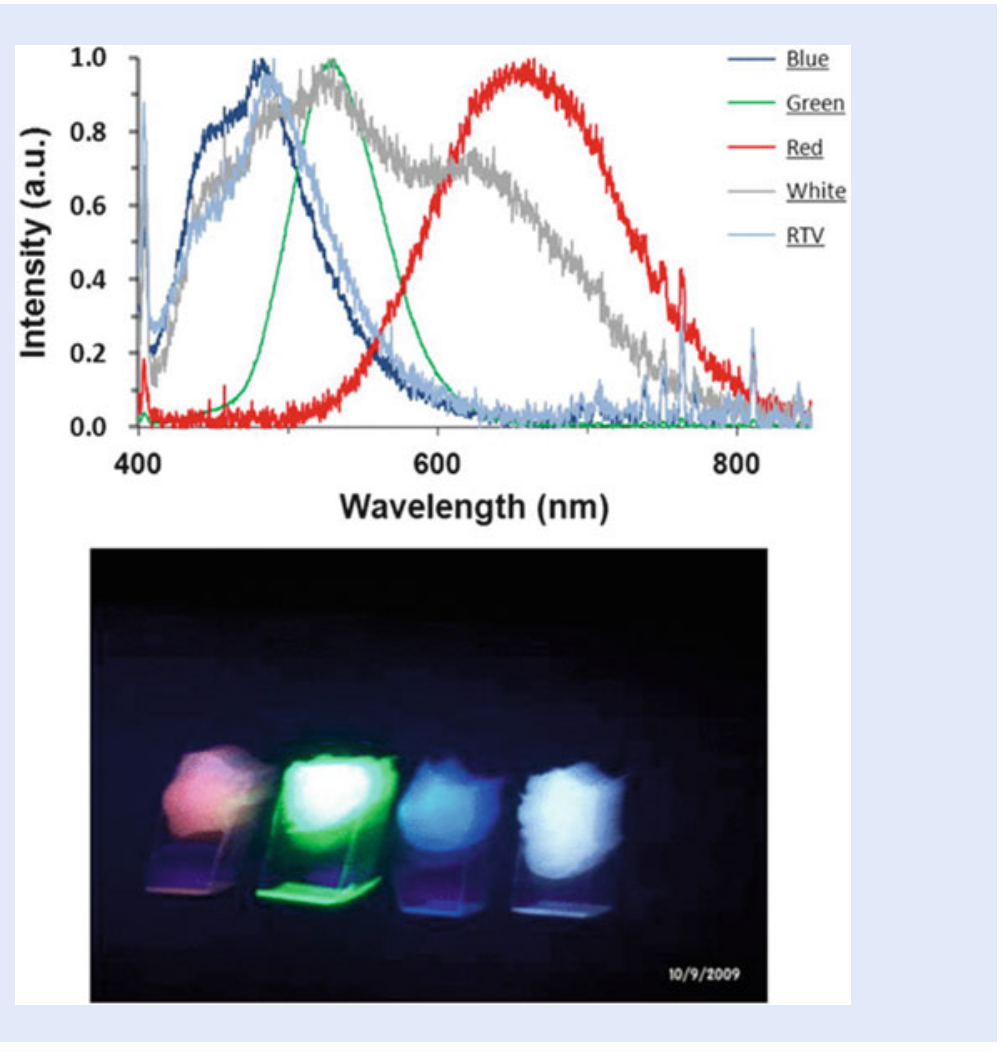

Fig. 10.28 Demonstration of 2.9-nm Si nanoparticles as equivalent to a "red phosphor" for LED technology. (Top) Normalized emission spectra of blue phosphor ZnS:Ag (in blue), green phosphor ZnS:Cu,Au,Al (in green) and red Si nanoparticles (in red) individually dispersed in RTV under the excitation of $365 \mathrm{~nm}$ radiation. The gray spectrum is due to a mixture of the three components dispersed in RTV under excitation of $365 \mathrm{~nm}$. Normalized spectrum of pure RTV (in light blue). (Bottom) from left to right the corresponding luminescent images of the individual components and of the mixture under excitation of $365 \mathrm{~nm}$ radiation

a near-infrared laser is used, which penetrates deep into the tissue, heating implanted nanoparticle to about $49^{\circ} \mathrm{C}$, as shown in - Fig. 10.29 [72]. This is the temperature level which is needed to kill many targeted cancer cells. This results in a threefold increase in killing cancer cells and a substantial tumor reduction within 30 days. Current hypothermic techniques are not selective, i.e., they involve applying heat to the whole body, which heats up cancer cells and healthy tissue, alike. Thus, healthy tissue tends to get damaged. By using gold nanoparticles, which amplify the low energy heat source efficiently, cancer cells can be targeted better and heat damage to healthy tissues can be minimized.

Improvement of the sensitivity of Raman analysis due to the enhanced electric field in the proximity of gold nanoparticles (as discussed above in $\bullet$ Sect. 10.4.2.1) has recently been used to monitor and confirm death of cancer cells. The extra sensitivity allows following changes in the molecular content inside cells, including destruction or formation of molecules in cancerous cells during their death [70].

\subsection{Plasmon Effect in Ancient Technology and Art}

Red glasses have been found in Italy that dated back to the late Bronze Age. The phenomena stayed essentially unsolved till the last decade. It is now believed that the colors are attributed to white light excitation of plasmon surface modes of electron oscillations in different metal nanoparticles incorporated during the 


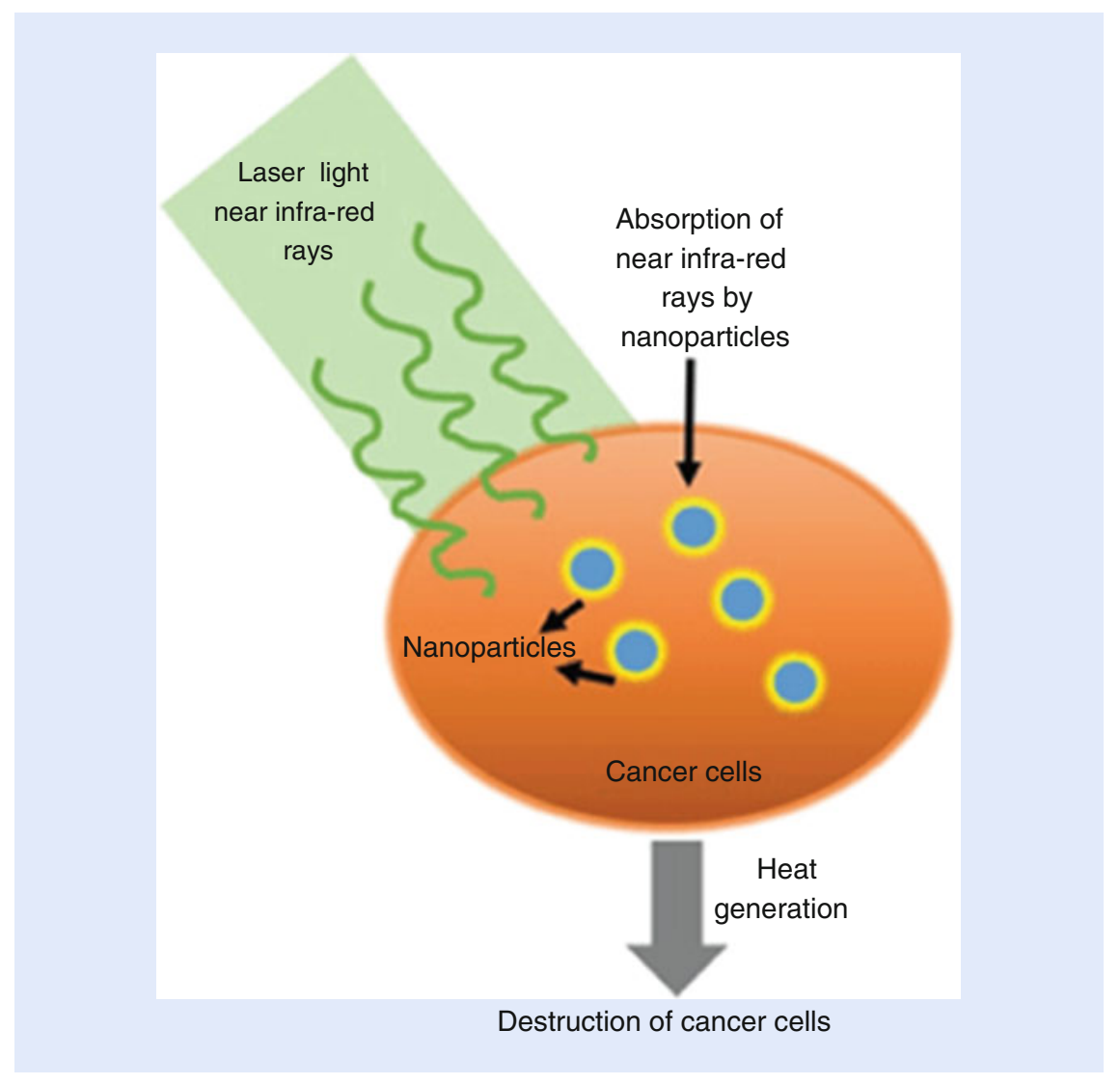

- Fig. 10.29 Schematic of plasmonic nanoparticle-based photothermal therapy for cancer treatment. A non-consequential weak infrared light gets concentrated in gold nanoparticles, heating them enough to kill cells (Adapted from [69])

manufacturing process $[73,74]$. In addition, gold nanoparticles were identified in some red tesserae of Roman times [75]. A vivid example is the well-known Roman Lycurgus Cup in glass dated from the fourth century CE and currently exhibited in the British Museum, as shown in - Fig. 10.30 (left) [76], and the elaborate and complex multicolor stained glass window shown in • Fig. 10.30 (right) [77].

As was discussed above in - Sect. 10.2.3, the phenomenon can be easily understood in terms of color mixing effects. When white light shines only the $G$ (green) component resonates with the electrons in the gold nanoparticles and is absorbed, hence removed. The B (blue) light is weakened by suffering scattering in all direction. The remaining $\mathrm{R}$ (red) component passes through. This is the reason that stained glass mixed with gold nanoparticles appears red to the naked eye.

In the Middle Ages exploitation of the outstanding optical properties of metallic nanoparticles became more sophisticated. Abbasid and Fatimid Islamic potters were able as early as $836-883 \mathrm{CE}$ to develop new chemical technology that allowed them to create complex nanometal-based multilayer structures. First they were incorporated in ceramics, which resulted in the emergence of lusterware, a special type of glazed ceramics, with striking optical effects. Secondly, the distribution of nanoparticles was programmed to include multilayers such that luster decoration color can change depending on the angle from which it is observed. An example of these types of ceramic decorations is given in - Fig. 10.31 [78]. The color change is spectacular and brilliant. A variety of very intense colored metallic shine including golden-yellow, blue, green, pink, etc., can be observed. Recent studies in 2005 concluded that the multilayer features give rise to light interference 


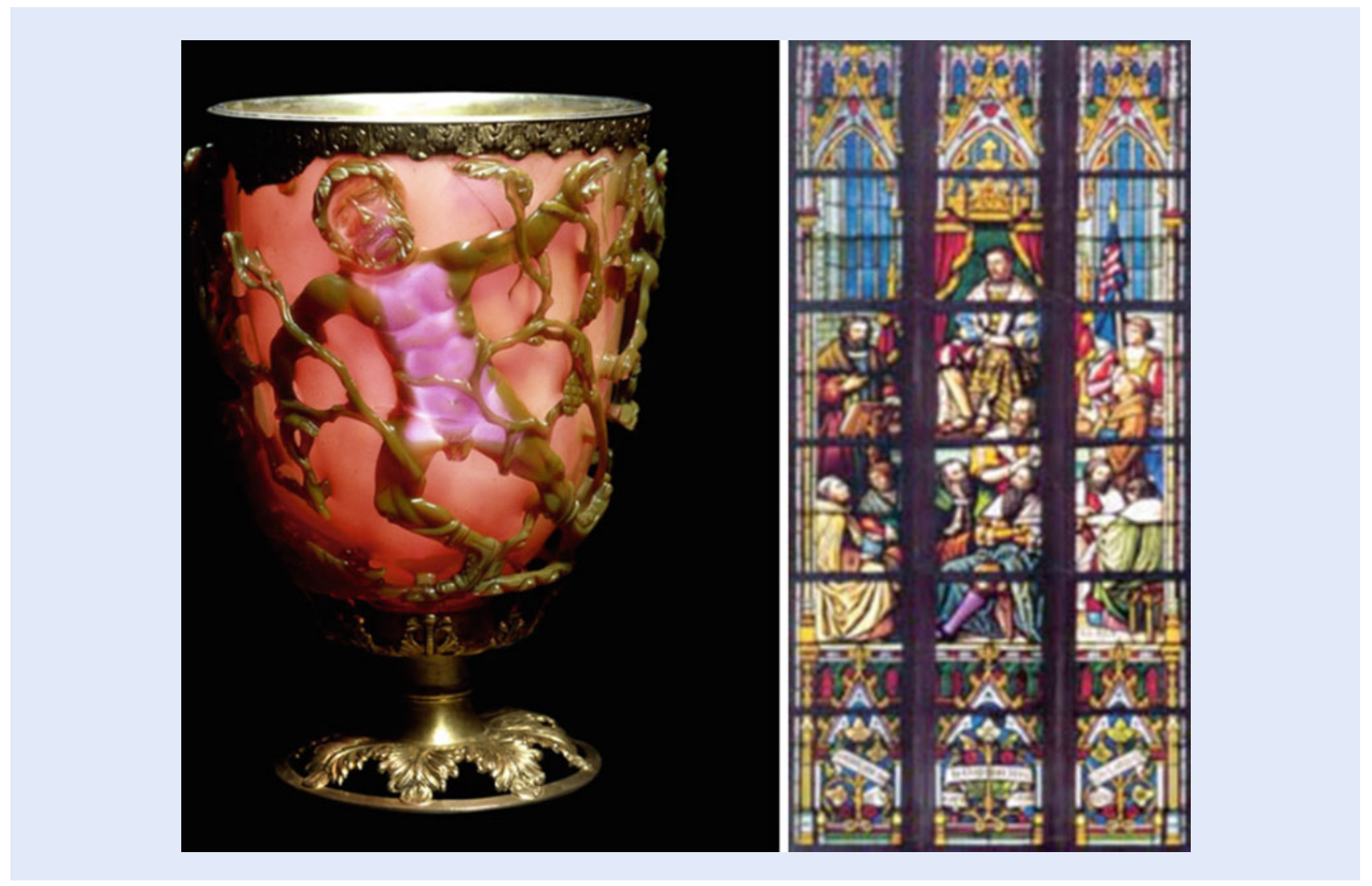

Fig. 10.30 (Left) The well-known Roman Lycurgus Cup in glass dated from the fourth century CE. It is currently exhibited in the British Museum (Right) Medieval stained glass windows (Courtesy: NanoBioNet and $\triangleright$ www.nano.gov] [76])

phenomena and scattering through rough interfaces, which adds to the surface plasmon effect and strongly contributes to the observed color [79].

Several material analysis studies [80-83] confirmed that the color of the luster decorations come from metallic nanoparticles. For instance, the presence of metal particles was directly confirmed by conducting high resolution transmission electron microscopy and imaging (TEM) along with material analysis using electron energy loss spectroscopy. Generally, copper and silver clusters were incorporated by applying a mixture of a paint, which contained copper and silver salt powders onto a glazed ceramic. This was followed by annealing in a reducing atmosphere. It is now believed that the basic mechanism involves the two processes of ion exchange and crystallization (nucleation and crystal growth) of copper and silver metallic nanoparticles inside the glassy matrix [84].

The ingenious technology, however, was way ahead of its time hence it was based on empirical chemical means, which made the technology vulnerable to extinction. In fact the technology has nowadays partially been lost. Vincent Reillon and collaborators [85] performed elaborate optical modeling, which confirmed the role of multilayer interference and interface scattering in the color of metallic shine reflection (specular direction). On the experimental side, modern artists [81, 83] conducted experiments to re-create luster decorations. They used modern kilns, which allowed alternative oxidization (oxygen flux) and reduction (CO flux) phases during the firing [73]. The best recreations achieved exhibited partial multilayer structure with a weaker organization. 

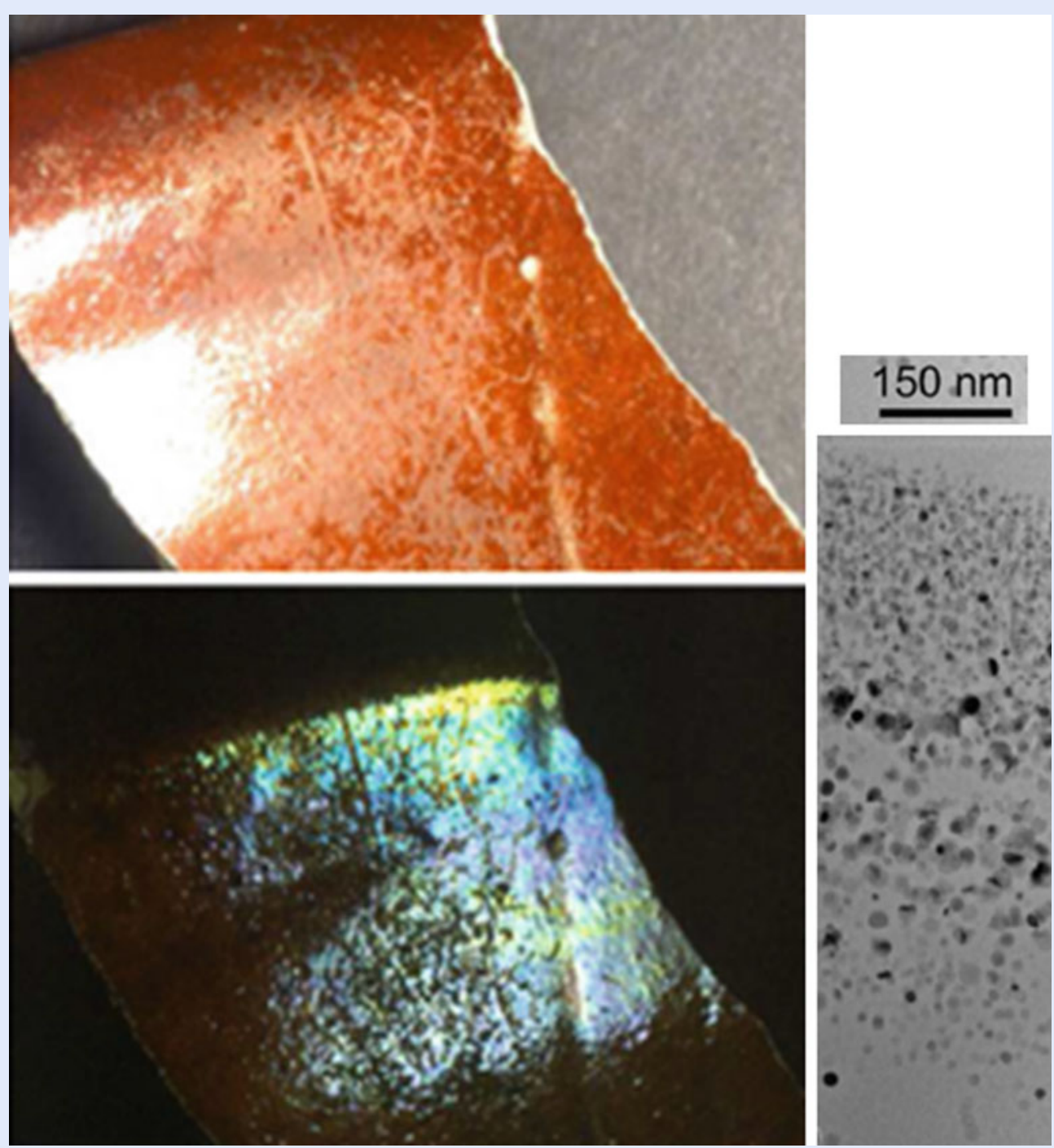

- Fig. 10.31 A nineth century CE lusterware from Mesopotamia (Susa). (Top left) Lusterware appears red. (Bottom left) It appears blue/green when viewed from different angle. (Micrographs, courtesy D. Chabanne), (right) TEM image showing the presence of metal nanoparticle (Adapted from $[77,78]$ )

\subsection{Alhasan Ibn Alhaytham (Alhazen) and the Nature of Light and Lusterware}

The Abbasid and Fatimid era are considered as two of most creative golden ages of Muslim civilization. The era saw many fascinating advances in science and technology. The period witnessed an aggressive and stimulating advanced chemical and manufacturing technology of glass and pottery, exploiting advanced lighting, color and optical effects of metal nanostructures produced from mixed silver and gold powders. Not only the chemical industry was advanced, material and metallurgical industry including thermo-mechanical forging and annealing was sophisticated. Steel cakes were subjected to such protocols to refine the steel to its exceptional quality. Domestic and military products were produced. One of the products of the industry, the famous Damascus blades, was pivotal in the fight against the Crusaders. Those were forged directly from small cakes of steel (named ' wootz') imported from ancient India $[86,87]$. The blades were remarkably hard, 
sharp, and light weight. In fact recent high resolution transmission electron microscopy of samples of the blades revealed the presence of carbon nanotube, which afforded the products exceptional material properties.

Alhasan Ibn Alhaytham (Alhazen) was born in Basra, Iraq in this flourishing era (965). He was not an exception; he contributed to several fields including astronomy, mathematics, medicine, and especially optics. He ventured into pioneering experimental scientific research, with methodology approaching what we use today in the finest research institutes. His studies examined the nature of light and color which resulted in several revolutionary breakthroughs. With limited instrumentation he embarked on thorough examination of the passage of light through various media and its dispersion into its constituent colors, which led him to challenge and refute several adopted notions in optics including:

- Constructing the first generation of pinhole cameras for experimental research

- Establishing, using his pinhole camera, that the human eye is not a source but a sensor or detector of light and color; and accurately described the mechanism of sight and the anatomy of the eye;

- Classifying light sources as luminescent and scattering sources [88, 89];

- Giving the first complete (unique) presentation of the law of reflection, by putting the incident ray and the reflected ray in the plane of incidence [90];

- Making the laws of refraction unique by introducing the pivotal principle of putting the incident and the refracted rays in the same plane (incidence plane) [90];

- Introducing the reciprocal law of refraction, which Kepler used to discover the law of total reflection [90];

- Proving light travels in straight line and that the speed of light is not infinite rather finite with different speeds in matter, moving more slowly in dense media, which was pivotal to the development of Fermat principle [91];

- Concluding that light is a mix of different colors by conducting experiments on the dispersion of light into its constituent colors; and the discovery that rainbows are caused by refraction of light; those were the first experiments to be conducted before the more detailed experiments conducted by Newton using advances in optical equipment, such as prisms to break up white light into a range of colors [92];

- Studying atmospheric refraction, and using it to discover that twilight only ceases or begins when the sun is $19^{\circ}$ below the horizon and to deduce the height of the atmosphere to be 55 miles, which is basically correct ( $\nabla \mathrm{http}: / /$ www.jackklaff.com/hos.htm);

- Laying the foundation for the scientific method [93];

- Writing the first comprehensive book on optics, which got translated into Latin, powering research in optics in Europe for many centuries;

These developments show that the quest of Alhazen revolutionized the study of optics at the time and laid the foundation for the scientific method [93]. Alhazen, however, did not try to solve the optical phenomenon of stained glass or lusterware. But those were problems for future generations. For centuries to follow, artists kept mixing silver and gold powder with glass or ceramics to fabricate colorful glass or pottery while no body was able to produce a scientific reason as to how these ingredients worked. The first step towards explaining the phenomenon had to wait until 1908 when Gustav Mie developed a theory of the optical properties of metallic colloids [94]. He showed that the color of a metal nanoparticle depends on its size as well as on the optical properties of the precursor metal and the adjacent dielectric materials. But full understanding of stained glass and lusterware required even more time and nineteenth to twenty-first century developments including electromagnetic theory, electromagnetic wave nature, and interference/diffraction and scattering of light, solid state theory, plasma 
theory, nanoscale phenomenon, advanced material characterization technologies, such as electron-based imaging and material analysis. In fact, it was only in the last two decades the scientific community has succeeded in solving the problem [95]. Those medieval artists or chemical industrialists were actually "nanotechnologists" synthesizing metal nanoparticles and harnessing what we today call plasmonics: a new field based on electron oscillations in metals called plasmon.

The recognition of the work of Alhazen may have been slow in the Middle Ages, but it has been coming strong in recent years. In medieval Europe, he was honored as Ptolemaeus Secundus ("Ptolemy the Second") or simply called "The Physicist." In the twentieth to twenty-first centuries he has been given several titles including "the First Scientist"; "Hero of Science"; "The Father of Modern Optics"; Pioneering Scientist; Pioneering Scientific Thinker, Rare Genius in Physical Research, The Optical Scientist, etc. In fact, year 2015 marks the 1000th anniversary since the appearance of the Alhazen's remarkable seven volume treatise on optics "Kitab al-Manazir." The year 2015 has also been adopted as the year of light and the United Nations through its arm UNESCO launched "2015 international year of light (IYL2015)" as a global initiative intended to raise awareness of how optical technologies promote sustainable development and provide solutions to worldwide challenges in energy, education, agriculture, communications, and health. One of the major scientific anniversaries that will be celebrated during the 2015 International Year of Light is the works on optics by Alhazen (Ibn Al-Haytham) (1015). UNESCO and the IYL2015 in partnership with the UK-based organization 1001 Inventions launched a high-profile international educational campaign celebrating Ibn al-Haytham. Moreover, 1001 Inventions and the King Abdulaziz Center for World culture in partnership with UNESCO and the IYL2015 are planning to produce a short film on his work. King Abdulaziz City for Science and Technology is also celebrating this occasion by publishing an edited book on optics for a very broad audience, to which this article is contributed.

\subsection{From Alhazen to Newton to the Trio: Dispersion of Light}

In 1015 Alhazen was concerned with understanding the different color components of light through natural phenomenon, such as propagation of light in material, reflection and refraction, and the rainbow effect. Newton 600 years later used manufactured glass prisms which became available then to deliberately disperse light into its components and to introduce the seven color names red, orange, yellow, green, blue, indigo, and violet for segments of the spectrum. Nearly 300 years after Newton Theodor "Ted" Hansch and Arthur Schawlow of Stanford University and John "Jan" Hall of the University of Colorado set out to achieve big strides in the quest to disperse light and isolate extremely narrow components using much more advanced electronics and optics capabilities, such as prisms, gratings, interference filters, spectrographs, and telescopic light expanders. But such a highly pure color components would have extremely weak intensity, which would necessarily require amplification if it is to be a practical light source. In 1972, the Stanford group dispersed the red fluorescence from a chemical dye into much finer components while simultaneously being amplified using the very advanced light-matter interaction concepts of stimulated emission (introduced by Einstein) and laser gain (Schawlow inventor) to produce a narrowband directed red laser (light amplification by stimulated emission of radiation). Pulses or flashes of red light of 8 ns durations with a record color definition (bandwidth) of $0.0004 \AA$ (or $0.001 \mathrm{~cm}^{-1}$ or $30 \mathrm{MHz}$ ) and energy of $\sim 1 \mathrm{~nJ}$ per pulse were achieved (Hansch laser). In addition to being narrow band, the laser allows for change 
(tuning) of wavelength with good control and precision, a pivotal property for matching (resonating with) and studying electronic structure of atoms and molecules [96]. John Hall, on the other hand, used a different approach by starting out with the red light of a helium-neon gas laser $[97,98]$. This red laser light at a given time is narrow band (very pure in color) but it is practically not as narrow over longer times because its frequency or wavelength drifts as a result of drift in ambient conditions (temperature, vibrations, etc.). Using advanced optical and electronic equipment he succeeded in making the frequency or wavelength practically stand still for long times. This high finesse light source is extremely useful but it is not tunable in wavelength.

The two groups used these advanced devices in which the color of light has been defined much more precisely to perform high resolution laser spectroscopy measurements of unprecedented accuracy and intrinsic physical interest. Jan Hall used his source to measure accurately the speed of light, allowing a re-definition of the SI meter. It should be mentioned here that the first version of the helium-neon laser was invented in 1960 by Ali Javan (Azerbajani born in Tehran, Iran) at Bell Labs [99]. However the laser produced invisible light at $1.15 \mu \mathrm{m}$. Only 18 months later in 1962, Javan's colleagues White and Rigden of Bell Lab constructed the visible He-Ne laser which is more exciting, captivating, and convenient [100].

The Stanford group utilized the tunability of the Hansch laser in a Doppler free technique that was introduced theoretically earlier by Russian scientists [101]. The technique allows the laser to be blind to all moving atoms in a gas sample and see only the ones that happen to be stationary along the direction of the red light beam, which unmasks the true structure of the atoms. - Figure 10.32 (left top) is a photo from a celebration at Stanford recognizing Schawlow and Hansch as California Scientist of the year for these developments and the use of the narrowband tunable laser in precise spectroscopic measurements. Issa Shahin, a doctoral student in the Stanford group, was involved in using the narrow bandband dye laser to study the structure of sodium and hydrogen [96]. Munir Nayfeh, a doctoral student under the superposition of Hansch and Schawlow reproduced a variation to the Jan Hall's stable He-Ne light source (• Fig. 10.32 left bottom) [103] and utilized it as a wavelength standard along with the narrowband red laser to unmask and measure the hyperfine structure and binding energy of the simplest of all atoms, the hydrogen atom, whose binding energy is the corner stone of all fundamental constants in nature, namely the Rydberg constant. The study achieved then a record accuracy of 9 parts per billion, which allowed the improvements of all other fundamental constants [104]. The importance of improving the accuracy of the Rydberg constant was evident in 1995 when, on the occasion of the 100 year anniversary of the top American Journal Physical Review, the journal listed this achievement as one of the seminal papers in the 100 year life of the Journal, as shown in - Fig. 10.32 (right) [102].

Alhazen and Newton and the Trio were concerned with breaking light into its frequency components; other scientists, on the other hand, focused on the time domain. Short pulses of light of duration of micro $\left(10^{-6}\right)$, nano $\left(10^{-9}\right)$, pico $\left(10^{-12}\right)$, femto $\left(10^{-15}\right)$, and now atto $\left(10^{-18}\right)$ seconds have been isolated. In this regard, we mention that the Egyptian-born Ahmad Zewail used femtosecond pulses of light to capture the very real-time dynamics inside molecules, studies that earned him in 1999 the Nobel Prize in chemistry [105]. In the last decade the interest ventured into the atto second regime, providing capabilities to make realtime observations of valence electron motion in solids [106].

Schawlow, Hansch, and Hall were recognized by Nobel Prizes. Arthur Schawlow received the 1981 Nobel Prize in Physics for his work on development of laser light. Theodor W. Hänsch and John Hall shared with Roy Glauber (for his 

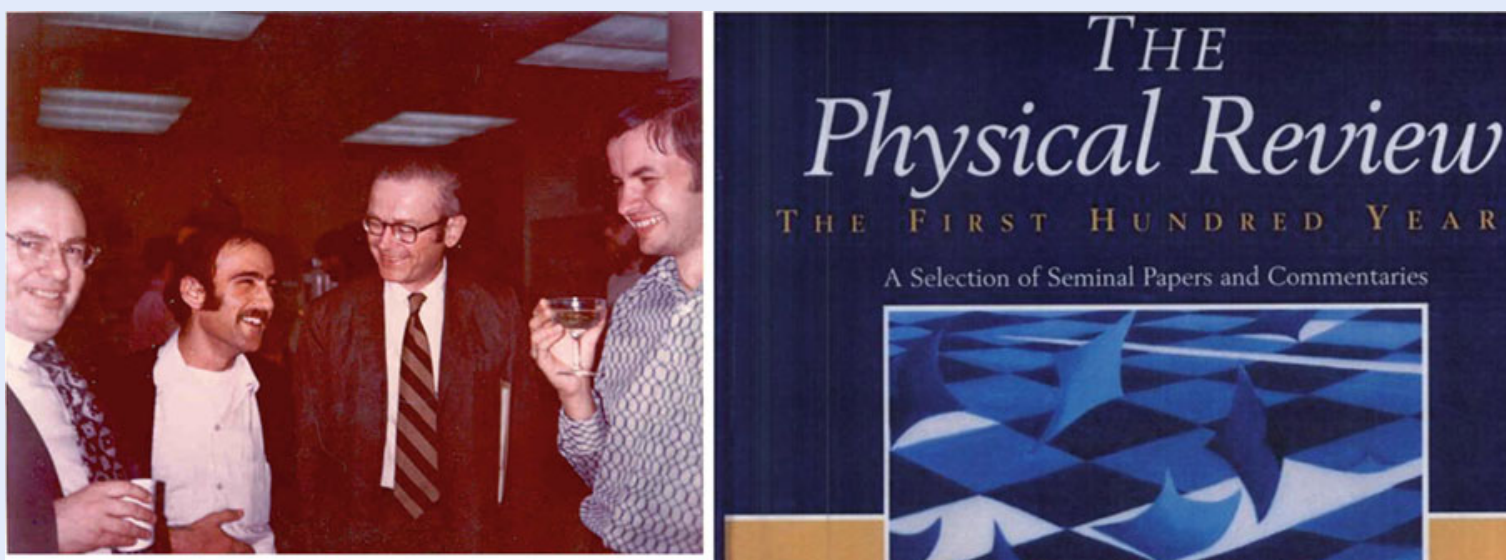

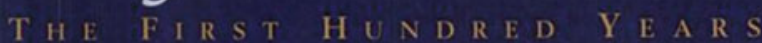

A Selection of Seminal Papers and Commentaries

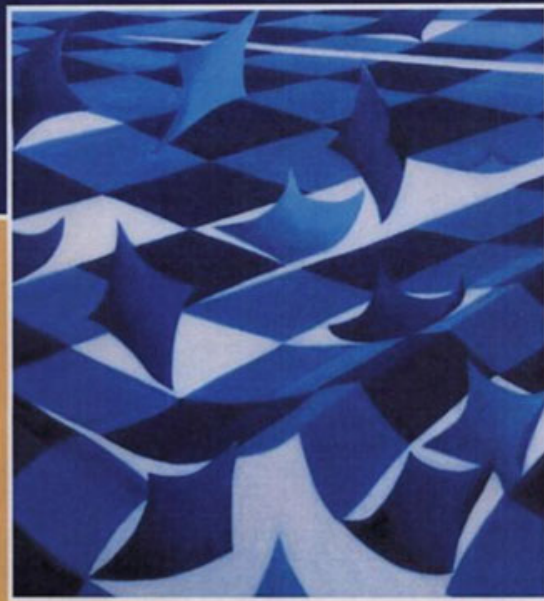

E D I TED B Y H. H E N R Y S T R O K E

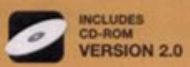

Fig. 10.32 (Left top) Celebration at Stanford from left to right: Arthur Schawlow, Munir Nayfeh, Stanford President, and Theodore Hansch for the choice of Schawlow and Hansch as California Scientist of the Year for the high resolution spectroscopy achievements. (Left bottom) Home built $\mathrm{He}-\mathrm{Ne}$ laser by Hansch and Nayfeh after [96] stabilized to 10 parts per billion. It has iodine vapor cell placed in the cavity. It is stabilized by locking it to the $n$th hyperfine component of isotropically pure $I^{129}$ at $633 \mathrm{~nm}$. The photo decorated the office of Arthur Schawlow (right) cover of the book celebrating the 100 year anniversary of the American Journal Physical Review [102]

work on coherence of light) the 2005 Nobel Prize in Physics for their work in precision spectroscopy using laser light sources.

It is to be noted that Newton's fame actually comes from gravity (as the father of) rather than from light. Alhazen fame is beginning to come in light, emerging as "the father of modern optics." There were many sources describing Ibn al-Haytham (Alhazen) as such. One of those is "Impact of Science on SocietyVolumes 26-27-(1976) Page 140, a prestigious UNESCO publication whose first edition came out in 1950. The study stated that "one name stands out as that of a rare genius in physical research: Abu Ali Al-Hasan Ibn Al-Haytham (965-1039) of Basra (Iraq), without question the father of modern optics" [107].

- Figure 10.33 presents selected clips and images from the mass media giving tribute to the contributions of Alhazen. There is one more thing that can be said about Alhazen. If the Nobel Prize was in place in the nineth century or its regulations did not exclude deceased recipients, this author believes Alhazen would have certainly been a recipient for his revolutionary breakthroughs in advancing our understanding of the nature of light. 


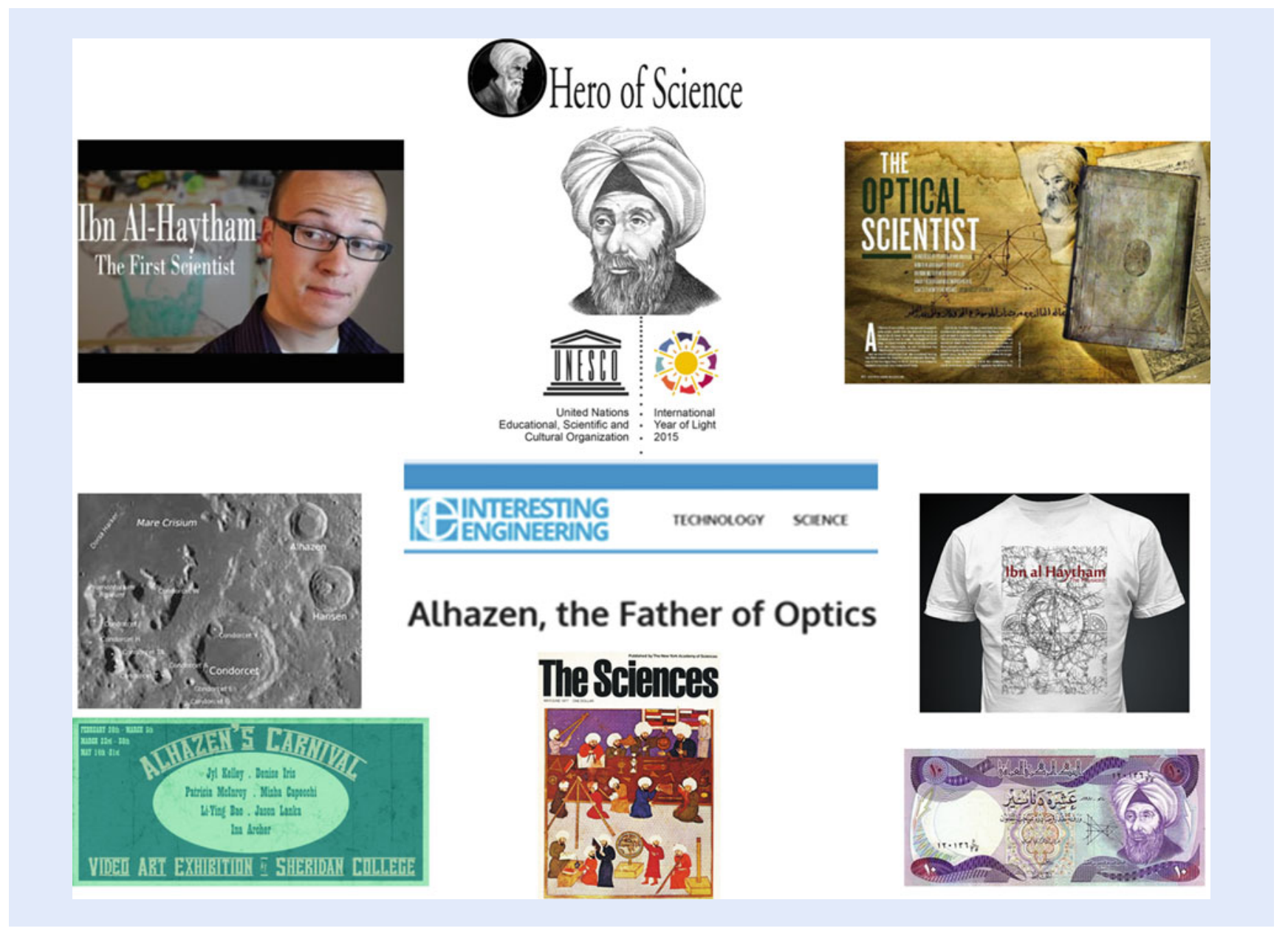

- Fig. 10.33 Selected clips and images from the mass media giving tribute to Alhazen contributions

\subsection{Conclusion}

We focused in this article on how miniaturization impacts light interaction and propagation in metal and semiconductors. Unlike semiconductors, coupling of light to oscillating free electrons in metals allows metal nanostructures to concentrate or focus light to spots limited only by their size, much beyond the wavelength of incoming light, which enables novel concepts of metal-based lenses. Moreover, strong concentration, absorption, and scattering induce drastic color change as well as temperature rise and interactivity with the surrounding environment. On the other hand, miniaturization of semiconductors affords the material strong size-dependent luminescence and color, to the degree that it can take indirect material, such as silicon from being the dullest material to a glowing material. Integration of plasmonic and semiconductor effects in hybrid architectures is promising synergetic applications.

These novel and unprecedented nanoscale optical functionalities enable a variety of exciting applications including a variety of futuristic applications [108]. But there are novel exciting applications which have been demonstrated and they may be around the corner for the consumer use, such as hyperthermia treatment of cancer, monitoring cell death through molecular changes, substance identification and detection, photovoltaic thin film solar cells, solid state white 
lighting, photodetectors, subwavelength waveguides and antennas for commercial, military and elementary particles applications, nanofabrication, integration of optics and electronics, and art of stained glass and lusterware.

Metal plasmonic devices face, however, significant challenges because of heat losses at visible and other high frequencies important for telecommunication. Such heat losses have seriously limited their practical use in electronics; but the heat effect was turned into an asset in the fight on acute disease at the cellular level.

Acknowledgment We acknowledge financial grants that supported our work on Nanoengineered light emitting silicon, including the University of Illinois, US National Science Foundation, US Army, US Office of Naval Research, State of Illinois, Grainger Foundation, Beckman Foundation, King Fahd University, King Saud University and King Abdulaziz City for Science and Technology, Sharp of America, and SunGen of Canada, and Saudi ARAMCO.

\section{- Occasion}

2015 has been declared as the International Year of Light and Light-based Technologies. This year celebrates many milestones in the history of optics starting from the 1000 year anniversary of Ibn Al-Haytham's achievements in optics among which is his great book on Light. King Abdulaziz City for Science and Technology in Riyadh, Saudi Arabia is celebrating this occasion by publishing an edited book on topics in optics for a very broad audience.

Open Access This chapter is distributed under the terms of the Creative Commons Attribution 4.0 International License (http://creativecommons.org/licenses/by/ 4.0/), which permits use, duplication, adaptation, distribution and reproduction in any medium or format, as long as you give appropriate credit to the original author(s) and the source, a link is provided to the Creative Commons license and any changes made are indicated.

The images or other third party material in this chapter are included in the work's Creative Commons license, unless indicated otherwise in the credit line; if such material is not included in the work's Creative Commons license and the respective action is not permitted by statutory regulation, users will need to obtain permission from the license holder to duplicate, adapt or reproduce the material.

\section{References}

1. Editor (1993) McGraw-Hill encyclopedia of science and technology, 5th edn. McGrawHill, New York, NY

2. Drexler KE (1986) Engines of creation: the coming era of nanotechnology. Doubleday, New York

3. Feynman R (2009) There's plenty of room at the bottom. Nat Nanotechnol 4:781. doi: 10.1038/nnano.2009.356. - http://www.nature.com/nnano/journal/v4/n12/full/nnano. 2009.356.html

4. National Nanotechnology Initiative (2016) What is nanotechnology? http://www. nano.gov/nanotech-101/what/definition; Benefits and applications, http://www. nano.gov/you/nanotechnology-benefits

5. lijima S, Ichihashi T (1993) Single-shell carbon nanotubes of 1-nm diameter. Nature 363:603-605

6. Nayfeh MH, Rogozhina E, Mitas L (2002) Silicon nanoparticles: next generation of ultrasensitive fluorescent markers. In: Baratron M-I (ed) Synthesis, in functionalization, and surface treatment of nanoparticles. American Scientific Publishers, Stevenson Ranch 
7. Nayfeh MH, Mitas L (2007) Silicon nanoparticles: new photonic and electronic material at the transition between solid and molecule. In: Kumar V (ed) Nanosilicon. Elsevier, Amsterdam

8. Lohse SE, Murphy CJ (2013) The quest for shape control: a history of gold nanorod synthesis. Chem Mater 25:1250-1261

9. Babak Nikoobakht B, El-Sayed MA (2003) Preparation and growth mechanism of gold nanorods (NRs) using seed-mediated growth method. Chem Mater 15:1957-1962

10. Murray CB, Kagan CR, Bawendi MG (2000) Synthesis and characterization of monodisperse nanocrystals and close-packed nanocrystal assemblies. Annu Rev Mater Res 30:545-610

11. Foresight Institute (2016) Applications of nanotechnology. https://www.foresight. org/nano/applications.html

12. Pines D, Bohm D (1952) A collective description of electron interactions: Il. Collective vs individual particle aspects of the interactions. Phys Rev 85:338

13. Messiah A (1999) Quantum mechanics. Dover, Minneola

14. Maier SA (2007) Plasmonics: fundamentals and applications. Springer, New York

15. Ditlbacher H, Hohenau A, Wagner D, Kreibig U, Rogers M, Hofer F, Aussenegg FR, Krenn JR (2005) Silver nano wires as surface plasmon resonators. Phys Rev Lett 95:257403

16. Hamamatsu Photonics KK (2016) Nanophotonics. http://www.hamamatsu.com/eu/ en/technology/innovation/nanophotonics/index.html

17. Stockman MI (2004) Nanofocusing of optical energy in tapered plasmonic waveguides. Phys Rev Lett 93:137404

18. Evlyukhin AB, Bozhevolnyi SI, Stepanov AL, Kiyan R, Reinhardt C, Passinger S, Chichkov BN (2007) Focusing and directing of surface plasmon polaritons by curved chains of nanoparticles. Opt Express 15:16667-16680

19. Yin L, Vlasko-Vlasov VK, Pearson J, Hiller JM, Hua J, Welp U, Brown DE, Kimball CW (2005) Sub wavelength focusing and guiding of surface plasmons. Nano Lett 5:1399-1402

20. Verslegers L, Catrysse PB, Yu Z, White JS, Barnard ES, Brongersma ML, Fan S (2009) Planar lenses based on nanoscale slit arrays in a metallic film. Nano Lett 9:235-238

21. Veselago VG (1968) The electrodynamics of substances with simultaneously negative values of $\varepsilon$ and $\mu$. Sov Phys Usp 10:509-514 (Russian text 1967)

22. West $P$, Ishii S, Naik G, Emani N, Boltasseva A (2010) Identifying low-loss plasmonic materials, SPIE Newsroom. - http://www.academia.edu/1037839/Identifying_low-loss_ plasmonic_materials

23. White Noise (2014) > http://whitenoise.kinja.com/dimensions-in-semiconductors-cansomething-be-zero-dim-1543310309

24. Bawendi M (2016) $>$ http://nanocluster.mit.edu/research.php

25. Trwoga PF, Kenyon AJ, Pitt CW (1998) Modeling the contribution of quantum confinement to luminescence from silicon nanoclusters. J Appl Phys 83:3791

26. Canham LT (1990) Silicon quantum wire array fabrication by electro-chemical and chemical dissolution of wafers. Appl Phys Lett 57:1046

27. Heinrich JL, Curtis CL, Credo GM, Kavanagh KL, Sailor MJ (1992) Luminescent colloidal Si suspensions from porous Si. Science 255:66

28. Yamani Z, Thompson H, AbuHassan L, Nayfeh MH (1997) Ideal anodization of silicon. Appl Phys Lett 70:3404

29. Yamani Z, Ashhab S, Nayfeh A, Nayfeh MH (1998) Red to green rainbow photoluminescence from unoxidized silicon nanocrystallites. J Appl Phys 83:3929

30. Ackakir O, Therrien J, Belomoin G, Barry N, Muller J, Gratton E, Nayfeh M (2000) Detection of luminescent single ultrasmall silicon nanoparticle using fluctuation spectroscopy. Appl Phys Lett 76:1857-1859

31. Belomoin G, Therrien J, Smith A, Rao S, Chaieb S, Nayfeh MH (2002) Observation of a magic discrete family of ultrabright Si nanoparticles. Appl Phys Lett 80:841

32. Nielsen D, Abuhassan L, Alchihabi M, Al-Muhanna A, Host J, Nayfeh MH (2007) Currentless anodization of intrinsic silicon powder grains: formation of fluorescent $\mathrm{Si}$ nanoparticles. J Appl Phys 101:114302

33. Mitas L, Therrien J, Twesten R, Belomoin G, Nayfeh MH (2001) Effect of surface reconstruction on the structural prototypes of ultrasmall ultrabright $\mathrm{Si}_{29}$ nanoparticles. Appl Phys Lett 78:1918

34. Allan G, Delerue C, Lannoo M (1996) Nature of luminescent surface states of semiconductor nanocrystallites. Phys Rev Lett 76:2961

35. Draeger EW, Grossman JC, Williamson AJ, Galli G (2003) Influence of synthesis conditions on the structural and optical properties of passivated silicon nanoclusters. Phys Rev Lett 90:167402

36. Sundholm D (2003) First principles calculations of the absorption spectrum of $\mathrm{Si}_{29} \mathrm{H}_{36}$. Nano Lett 3:847 
37. Lehtonen O, Sundholm D (2005) Density-functional studies of excited states of silicon nanoclusters. Phys Rev B 72:085424

38. Ball $P$ (2001) Let there be light. Nature 409:974

39. Smith A, Yamani Z, Turner J, Habbal S, Granick S, Nayfeh MH (2005) Observation of strong direct-like oscillator strength in the photoluminescence of $1 \mathrm{~nm}$ silicon nanoparticles. Phys Rev B 72:205307

40. Mantey K, Zhu A, Boparai J, Nayfeh M, Marsh C, Alchaar G (2012) Observation of linear solid-solid phase transformation in silicon nanoparticles. Phys Rev B 85:085417

41. Rao S, Mantey K, Therrien J, Smith A, Nayfeh M (2007) Molecular behavior in the vibronic and excitonic properties of hydrogenated silicon nanoparticles. Phys Rev B 76:155316

42. Nayfeh M, Akcakir O, Belomoin G, Barry N, Therrien J, Gratton E (2000) Second harmonic generation in microcrystallite films of ultrasmall Si nanoparticles. Appl Phys Lett 77:4086

43. Kanemitsu Y (2003) Luminescence from Si/Si02 nanostructures. In: Pavesi L et al (eds) Towards the first silicon laser. Kluwer Academic, Dordrecht, pp 109-122

44. Pavesi L, Dal Negro L, Mazzoleni C, Franzò G, Priolo F (2000) Optical gain in silicon nanocrystals. Nature 408:440-444

45. Nayfeh MH, Barry N, Therrien J, Akcakir O, Gratton E, Belomoin G (2001) Stimulated blue emission in reconstituted films of ultrasmall silicon nanoparticles. Appl Phys Lett 78:1131

46. Nayfeh MH, Chaieb S, Rao S, Barry N, Therrien J, Belomoin G, Smith A (2002) Observation of laser oscillation in aggregates of ultrasmall silicon nanoparticles. Appl Phys Lett $80: 121$

47. Hilliard JE, Nayfeh HM, Nayfeh MH (1995) Re-establishment of photoluminescence in Cu quenched porous silicon by acid treatment. J Appl Phys 77:4130

48. Cho C-H, Aspetti CO, Park J, Agarwal R (2013) Silicon coupled with plasmon nanocavities generates bright visible hot luminescence. Nat Photonics 7:285-289

49. Gu Z, Liu S, Sun S, Wang K, Lyu Q, Xiao S, Song Q (2015) Photon hopping and nanowire based hybrid plasmonic waveguide and ring-resonator. Nat Sci Rep 5:917

50. Piccione B, Aspetti CO, Cho C-H, Agrawal R (2014) Tailoring light-matter coupling in semiconductor and hybrid-plasmonic nanowires. Rep Prog Phys 77:086401

51. Nicholaides C, Nayfeh MH, Clark CW (eds) (1989) Atoms in strong fields. Plenum, New York

52. Glab W, Nayfeh MH (1985) Stark induced resonances in the photoionization of hydrogen. Phys Rev A 31:530-532

53. Nedyalkov N, Imamova S, Atanasov P, Obara M (2010) Gold nanoparticles as nanoheaters and nanolenses in the processing of different substrate surfaces. J Phys Conf Ser 223:012035

54. Clery D (1992) Nanotechnology rules, OK! New Sci 1811:42

55. Yau S-T, Saltz D, Nayfeh MH (1990) Laser-assisted deposition of nanometer structures using scanning tunneling microscopy. Appl Phys Lett 57:2913

56. Hurst GS, Nayfeh MH, Young JP (1977) One-atom detection using resonance ionization spectroscopy. Phys Rev A15:2283

57. Lubkin G (1977) Resonance electron spectroscopy detects single atoms. Phys Today 30:17

58. Quanta (1977) One-atom chemistry. The Sciences. The New York Academy of Sciences, Quanta, p 5, May/June1977

59. Stupca M, Alsalhi M, Al Saud T, Almuhanna A, Nayfeh MH (2007) Enhancement of polycrystalline silicon solar cells using ultrathin films of silicon nanoparticle. Appl Phys Lett 91:063107

60. Maximenko Y, Elhalawany N, Yamani Z, Yau S-T, Nayfeh MH (2013) Polyaniline - Si nanoparticles nanocapsules as a dual photovoltaic sensitizer. Mater Res Soc Symp Proc 1500

61. Chowdhury Fl, Nayfeh MH, Nayfeh AM (2016) Enhancened performance of thin film silicon solar cells with a top film of silicon nanoparticles due to down-conversion and near resonance charge transport. J Sol Energy 125:332-338

62. Nayfeh M, Rao S, Nayfeh O, Smith A, Therrien J (2005) UV photodetectors with thin film Si nanoparticle active mediaum. IEEE Trans Nanotechnol 4:660

63. Magill S, Xie J, Nayfeh M, Yu H, Fizari M, Malloy J, Maximenko Y (2015) Enhanced UV light detection using wavelength-shifting properties of Silicon nanoparticles. J Instrum 10: P05008

64. Jee S-W, Zhou K, Kim D-W, Lee J-H (2014) A silicon nanowire photodetector using Au plasmonic nanoantennas. Nano Convergence 1:29

65. Qiu T, Wu XL, Mei YF, Wan GJ, Chu PK, Siu GS (2005) From Si nanotubes to nanowires: synthesis, characterization, and self-assembly. J Cryst Growth 277:143 
66. Mantey K, Shams S, Nayfeh MH, Nayfeh O, Alhoshan M, Alrokayan S (2010) Synthesis of wire-like silicon nanostructures by dispersion of SOI using electroless etching. J Appl Phys 108:124321

67. Catchpole KR, Polman A (2008) Plasmonic solar cells. Opt Express 16: 21793-21800 \& http:// www.opticsinfobase.org/oe/abstract.cfm?URI=oe-16-26-21793

68. Song H, Lee S (2007) Red light emitting solid state hybrid quantum dot-near-UV GaN LED devices. Nanotechnology 18:255202

69. Stupca M, Nayfeh OM, Hoang T, Nayfeh MH, Alhreish B, Boparai J, Aldwayyan A, AISalhi M (2012) Silicon nanoparticle-ZnS nanophosphors for UV- based white LED. J Appl Phys 112:074313

70. Kirui DK et al (2013) Targeted near-IR hybrid magnetic nanoparticles for in vivo cancer therapy and imaging. Nanomed Nanotechnol Biol Med 9:702-711

71. Kang B, Mackey MA, El-Sayed MA (2010) Nuclear targeting of gold nanoparticles in cancer cells induces DNA damage, causing cytokinesis arrest and apoptosis. J Am Chem Soc 132:1517-1519

72. Chen J, Keltner L, Christophersen J, Zheng F, Krouse M et al (2002) New technology for deep light distribution in tissue for phototherapy. Cancer J 8:154-163

73. Angelini I, Artioli G, Bellintani P, Diella V, Gemmi M, Polla A, Rossi A (2004) Chemical analyses of bronze age glasses from Frattesina di Rovigo, northern Italy. J Archaeol Sci 31:1175-1184

74. Artioli G, Angelini I, Polla A (2008) Crystals and phase transitions in protohistoric glass materials. Phase Transit 81:233-252

75. Colomban P, March G, Mazerolles L, Karmous T, Ayed N, Ennabli A, Slim H (2003) Raman identification of materials used for jewelry and mosaics in Ifriqiya. J Raman Spectrosc 34:205-213

76. Freestone I, Meeks N, Sax M, Higgitt C (2007) The Lycurgus cup - a Roman nanotechnology. Gold Bull 40:270-277

77. Mallmann M (2008) Medieval stained glass window. Science in School. Courtesy: NanoBioNet and, - www.nano.gov. - http://www.scienceinschool.org/2008/issue10/ nanotechnology

78. Mirguet C, Roucau C, Sciau P (2009) Transmission electron microscopy a powerful means to investigate the glazed coating of ancient ceramics. J Nano Res 8:141-146

79. Chabanne D (2005) Le décor de lustre métallique des céramiques glaçurées (IXèmeXVIlème siècles), Matériaux, couleurs et techniques. PhD thesis, University Bordeaux 3

80. Bobin O, Schvoerer M, Miane JL, Fabre JF (2003) Colored metallic shine associated to luster decoration of glazed ceramics: a theoretical analysis of the optical properties. J Non Cryst Solids 332:28-34

81. Colomban P (2009) The use of metal nanoparticles to produce yellow, red and iridescent color, from bronze age to present times in luster pottery and glass: solid state chemistry, spectroscopy and nanostructure. J Nano Res 8:109-132

82. Lafait J, Berthier S, Andraud C, Reillon V, Boulenguez J (2009) Physical colors in cultural heritage: surface plasmons in glass. C R Phys 10:649-659

83. Cizer S (2010) History, technique and art of Luster. Dokuz Eylul University, Narhdere, Izmir

84. Roqué J, Molera J, Cepria G, Vendrell-Saz M, Perez-Arantegui J (2008) Analytical study of the behaviour of some ingredients used in luster ceramic decorations following different recipes. Phase Transit 81:267-282

85. Reillon V, Berthier S (2006) Modelization of the optical and colorimetric properties of lustered ceramics. Appl Phys Mater Sci Process 83:257-265

86. Sanderson S (2006) Materials: carbon nanotubes in an ancient Damascus sabre, sharpest cut from nanotube sword. Nature 444:286

87. Reibold M, Paufler P, Levin AA, Kochmann W, Pätzke N, Meyer DC (2006) Materials: carbon nanotubes in an ancient Damascus sabre. Nature 444:286

88. Simon G (1996) Vision according to Alhazen: Sciences et savoirs aux XVle et XVlle siècles. Presses universitaires du Septentrion: 15

89. Le Guet Tully F (2012) Brief history of astronomical optics. - https://lise.oca.eu/spip. php?rubrique37

90. Mach E (2003) The principles of physical optics: an historical and philosophical treatment. \ https://books.google.com/books?isbn $=0486495590$

91. Høg E (2008) 650 Years of optics: from Alhazen to Fermat and Rømer, Astrometry and optics during the past 2000 years - arXiv.org. $>$ https://arxiv.org/pdf/1104.4554. www. astro.ku.dk/ erik/HoegAlhazen.pdf

92. Color Spaces - color phenomena (2010) $\downarrow$ www.color-theory-phenomena.nl/08.00.html

93. Toler P (2012) Alhazen: the first true scientist? Wonders and Marvels. $>$ http://www. wondersandmarvels.com/2012/08/alhazan-the-first-true-scientist.html 
94. Mie G (1908) Beiträge zur optik trüber medien, speziell kolloidaler metallösungen. Ann Phys 25:377-445

95. Wagner H-P, Kaveh-Baghbadorani M (2015) Plasmonics: revolutionizing light-based technologies via electron oscillations in metals. http://phys.org/news/2015-06plasmonics-revolutionizing-light-based-technologies-electron.html

96. Hänsch TW, Shahin IS, Schawlow AL (1971) High-resolution saturation spectroscopy of the sodium D lines with a pulsed tunable dye laser. Phys Rev Lett 27:707

97. Baer T, Kowalski FV, Hall JL (1980) Frequency stabilization of a $0.633-\mu \mathrm{m} \mathrm{He}-\mathrm{Ne}$ longitudinal Zeeman laser. Appl Opt 19:3173-3177

98. Schweitzer WG Jr, Kessler EG Jr, Deslattes RD, Layer HB, Whetstone JR (1973) Description, performance, and wavelengths of iodine stabilized lasers. Appl Opt 12:2927

99. Javan A, Herriott D, Bennett W (1961) Population inversion and continuous optical maser oscillation in a gas discharge containing a He-Ne mixture. Phys Rev Lett 6:106

100. White AD, Rigden JD (1962) Continuous gas maser operation in the visible. Proc IRE 50:1697

101. Vasilenko LS, Chebotayev VP, Shishaev AV (1970) JETP Lett 12:113

102. Stroke HH (ed) (1995) The physical review: the first 100 years: a selection of seminal papers and commentaries. Springer/AIP press, New York

103. Nayfeh MH (1974) Precision measurement of the Rydberg by saturated spectroscopy. PhD thesis, Stanford University

104. Hänsch TW, Nayfeh MH, Lee SA, Curry SM, Shahin IS (1976) Precision measurement of the Rydberg constant by laser saturation spectroscopy of the Balmer line in hydrogen and deuterium. Phys Rev Lett 32:1336

105. Zewail AH (1990) The birth of molecules. Sci Am 263:76

106. Reiter F, Graf U, Serebryannikov EE, Schweinberger W, Fiess M, Schultze M, Azzeer AM, Kienberger R, Krausz F, Zheltikov AM, Goulielmakis E (2010) Route to attosecond nonlinear spectroscopy. Phys Rev Lett 105:243902-243904

107. UNESCO (1976) Impact Sci Soc 26-27:140

108. Focus/Feature (2015) Nano-optics gets practical. Nat Nanotechnol 10:11-15 